Madrid, Spain

24-26 June 2009

Published: 23 June 2009

(C) 2009 BioMed Central Ltd
Speaker Abstracts

\section{SYMPOSIUM I}

\section{Advances in prevention, diagnosis and genetics}

\section{S1}

\section{Identifying and assessing women at high risk for breast cancer \\ JE Garber}

Dana Farber Cancer Institute, Boston, MA, USA

Breast Cancer Research 2009, 11(Suppl 1):S1 (doi: 10.1186/bcr2262)

There has been progress in the identification of factors that confer important risk for the development of breast cancer. The factors include: heritable mutations in susceptibility genes; exposure to therapeutic radiation during breast development (as for Hodgkin's disease survivors who received therapeutic radiation to the chest); and histologic lesions, including LCIS and atypical hyperplasias.

Testing for mutations in the $B R C A 1$ and $B R C A 2$ breast ovarian cancer susceptibility genes has become part of the established care of breast cancer patients. Genetic information from BRCA1/2 testing is used to help healthy at-risk women to avoid breast and/or ovarian cancer, and ultimately to avoid death from those cancers. Data accumulated over the past decade have provided evidence that breast cancer surveillance can be improved with the addition of breast MRI, that prophylactic oophorectomy substantially reduces the risk of ovarian cancer and, when performed before menopause, can reduce the risk of breast cancer as well, and that prophylactic mastectomy reduces the risk of breast cancer by more than $90 \%$.

It has been observed that approximately $80 \%$ of BRCA1-associated breast cancers are negative for ER, PR and HER2 (so-called triple negative) and cluster with basal-like breast cancers by DNA microarray, while $80 \%$ of BRCA2-associated breast cancers are $\mathrm{ER}^{+}$and $\mathrm{PR}^{+}$, but HER2 negative, and luminal. These data are surprising given the close relationships between these genes in their DNA repair activities, and raise some concern that hormonal interventions will not successfully reduce the risk of $B R C A 1$-associated breast cancers. Other strategies may be necessary to reduce breast cancer risk for this group.

Genetic information has been shown to have important implications for women with breast cancer as well. Women with strong family histories of breast and/or ovarian cancer, and women diagnosed before age 40 may consider testing at the time of breast cancer diagnosis if they would use the information to make treatment decisions. Some women choose bilateral mastectomies over breast-conserving treatment if they learn that their risk of second primary breast cancer exceeds $50 \%$, and if their prognosis from the original breast cancer is good. Some women opt for oophorectomy as part of the management of their $\mathrm{ER}^{+}$breast cancer if they are premenopausal mutation carriers (and could participate in TEXT).
Recently, novel agents, the PARP inhibitors, have been shown to be effective in the phase II trials in women with BRCA1 or BRCA2 mutations and metastatic ovarian or breast cancer. These drugs target DNA repair pathways that are particularly vulnerable in women with $B R C A 1 / 2$ mutations. The agents may also be effective in women with sporadic breast cancer, and are currently in trials in Europe and the United States alone and in combination with cytotoxic agents.

\section{S2}

Magnetic resonance imaging for diagnosis, staging, and follow-up

\section{Morrow}

Memorial Sloan-Kettering Cancer Center, New York, NY, USA

Breast Cancer Research 2009, 11(Suppl 1):S2 (doi: 10.1186/bcr2263)

There is considerable debate regarding the role of magnetic resonance imaging (MRI) in the management of the breast cancer patient. MRI should not be used as a diagnostic test to exclude the presence of carcinoma. In one multi-institutional study of 1,004 women, the positive predictive value of MRI was $72 \%$, and the overall sensitivity $88 \%$ [1]. Recognition that MRI identifies additional areas of cancer not detected by other imaging modalities in an average of $16 \%$ of breast cancer cases [2] has led to great interest in its use to select women for breastconserving surgery (BCS). Suggested benefits of MRI include a reduction in margin positivity and conversion from BCS to mastectomy, and a decrease in local recurrence rates. Several retrospective studies and one prospective randomized [3] trial have addressed the impact of MRI on the short-term surgical outcomes. These studies have uniformly failed to demonstrate a benefit for MRI. In the prospective randomized Comparative Effectiveness of Magnetic Resonance Imaging in Breast Cancer (COMICE) trial, re-excision was required in $10 \%$ of the MRI group and $11 \%$ of the non-MRI group, with conversion to mastectomy in $6 \%$ and $8 \%$, respectively. Most studies show that MRI approximately doubles the overall likelihood of undergoing mastectomy without decreasing unplanned mastectomy. Solin and colleagues examined the effect of MRI on local recurrence after BCS with radiation therapy (RT) and on contralateral cancer [4]. At 8 years the incidence of contralateral cancer was $6 \%$ in both the MRI and non-MRI groups, and local recurrence was seen in $3 \%$ of those with an MRI at diagnosis and in $4 \%$ of those without. The repeated observation that MRI finds two to four times as much disease as becomes evident as local recurrence indicates that the majority of this disease is controlled with RT. In addition, the existence of local recurrence after mastectomy indicates that some local recurrence is a manifestation of biologically aggressive disease (first site of metastases), which is unlikely to be influenced by the use of MRI. Current indications for the use of MRI in patients with breast cancer include: known or suspected $B R C A 1 \& 2$ mutation carriers who choose not to undergo bilateral mastectomy; patients presenting with metastases to the axillary nodes and no evident breast tumor; patients with Paget's disease of the nipple and no evident breast tumor; or the uncommon patient with a major discrepancy between clinical findings and the results of mammography and ultrasound. 
A benefit for MRI in the follow-up of the breast cancer patient is also unproven. Local recurrence after BCS and RT is uncommon, occurring in fewer than $8 \%$ of patients at 10 years. The size of the local recurrence is not a prognostic factor, and the idea that early detection will improve prognosis does not reflect the biology of local recurrence. The treatment of local recurrence regardless of size is mastectomy, and salvage mastectomy results in local control in 85 to $95 \%$ of patients. These good outcomes must be weighed against the cost of MRI, the high rate of short-interval follow-up and biopsies generated, and the lack of a clear rationale for using the examination for follow-up.

\section{References}

1. Bluemke DA, Gatsonis CA, Chen MH, DeAngelis GA, DeBruhl N, Harms S, Heywang-Köbrunner SH, Hylton N, Kuhl CK, Lehman C, et al:: Magnetic resonance imaging of the breast prior to biopsy. JAMA 2004, 292:2735-2742.

2. Houssami N, Ciatto S, Macaskill P, Lord SJ, Warren RM, Dixon JM, Irwig L: Accuracy and surgical impact of magnetic resonance imaging in breast cancer staging: systematic review and metaanalysis in detection of multifocal and multicentric cancer. J Clin Oncol 2008, 26:3248-3258.

3. Drew PJ, Harvey I, Hanby A, Brown S, Olivier C, Napp V, Brown J, Turnbull LW, on Behalf of Comice Trialists: The UK NIHR multicentre randomised COMICE trial of MRI planning for breast conserving treatment for breast cancer. Cancer Res 2009, 69(2 Suppl):Abstract 51.

4. Solin LJ, Orel SG, Hwang WT, Harris EE, Schnall MD: Relationship of breast magnetic resonance imaging to outcome after breast-conservation treatment with radiation for women with early-stage invasive breast carcinoma or ductal carcinoma in situ. J Clin Oncol 2008, 26:386-391.

\section{S3}

\section{Genetic profile sets: differences and preferences $M$ van de Vijver}

Department of Pathology, Academic Medical Center, Amsterdam, the Netherlands

Breast Cancer Research 2009, 11(Suppl 1):S3 (doi: 10.1186/bcr2264)

High-throughput genetic analysis of breast cancer results in improved classification of breast cancer and can be used to identify prognostic and predictive factors. These prognostic and predictive factors are increasingly useful to guide therapy decisions in individual breast cancer patients.

There is a rapidly increasing body of literature on gene expression signatures associated with prognosis; and on gene expression signatures associated with response to specific systemic treatment, including chemotherapy, hormonal therapy and HER2 targeted therapy. The main prognostic profiles published to date are a 70-gene signature identified by our group in the Netherlands Cancer Institute (Amsterdam, the Netherlands); a 76-gene prognosis profile identified by investigators from Erasmus Medical Centre (Rotterdam, the Netherlands; a 21-gene recurrence score developed by the company Genomic Health (Redwood City, USA); and a genomic-grade signature developed by investigators at the Institut Bordet (Brussels, Belgium).

Breast cancer is a very heterogeneous disease, and prognostic and predictive profiles may differ in breast cancer subtypes. We are therefore currently performing gene expression profiling studies, assessment of DNA copy number changes and miRNA expression profiles in triplenegative breast cancers $(n=96)$ and HER2-positive breast cancers $(n=140)$.

Prognostic classifiers can be used to identify patients that will benefit most from adjuvant systemic therapy, but other classifiers will be needed to decide which treatment should be given.

To guide the choice of chemotherapy, hormonal therapy and targeted therapy, neoadjuvant studies are well suited to identify predictive factors for therapy response. For this purpose, we have analysed gene expression profiles in pretreatment biopsies of 191 patients treated with neoadjuvant chemotherapy; and patients with HER2-positive trastuzumab. Our results and studies from various other groups show that basal-type/triple-negative tumors show a pathological complete remission in 30 to $40 \%$ of cases; as compared with $<5 \%$ in luminaltype tumors. It has been more difficult to identify gene expression profiles associated with response to chemotherapy and response to trastuzumab using supervised classification techniques. Research aimed at the identification of genetic classifiers for responsiveness to specific systemic therapies is expanding rapidly and should lead to clinically useful tests in the coming years.

At present, there are several ongoing randomised clinical trials investigating genetic profiling in guiding adjuvant systemic therapy; and in neoadjuvant systemic therapy. These studies will enable us to better understand differences between genetic sets; and will allow us to develop our preferences based on results obtained in large well-controlled trials.

\section{S4}

\section{Molecular heterogeneity of breast cancer: implications for treatment and clinical trial design \\ L Pusztai}

Department of Breast Medical Oncology, The University of Texas MD Anderson Cancer Center, Houston, TX, USA

Breast Cancer Research 2009, 11(Suppl 1):S4 (doi: 10.1186/bcr2265)

Comprehensive molecular analysis of breast cancer indicates that breast carcinoma is not a single disease with heterogeneous morphology and variable hormone receptor expression, but a collection of molecularly distinct neoplastic diseases of the breast. The magnitude of molecular differences that are seen between estrogen receptor (ER)-positive and ER-negative cancers at the mRNA expression or the DNA copy number level are similar to the extent of molecular differences that distinguish acute myeloid leukemias from lymphoid leukemias. The clinical differences between ER-negative and ER-positive cancers have long been recognised and the recent genomic data provided further evidence that these cancers represent different diseases. Furthermore, two major groups within the ERpositive cancers can also be easily recognised at the molecular level: one that corresponds to high-grade, highly proliferative tumors; and the other to lower-grade cancers with low proliferation rate. These two molecular subsets have very different prognosis: the former group is less sensitive to endocrine therapy and has poor prognosis but is more sensitive to cytotoxic drugs, and therefore adjuvant chemotherapy may improve outcome. The latter group has excellent prognosis with endocrine therapy alone, and does not appear to benefit much from adjuvant chemotherapy. Several different molecular assays can assist in distinguishing between these different prognostic subsets of ERpositive cancers; the most commonly used commercial test in the United States is Oncotype DX; however, the MammaPrint and GenomicGrade molecular assays are also able to identify ER-positive patients with excellent prognosis with endocrine therapy. In the absence of the statistically more accurate multivariate molecular prediction models, the histologic grade, HER2 status and Ki67 may be used as a poor man's alternative to estimate prognosis and chemotherapy sensitivity of ER-positive cancers. The recognition that breast cancer is not a single disease has important consequences for clinical trialists and academic investigators. Different diseases require separate clinical trials, and different biomarkers may be needed to predict response or prognosis accurately in the different disease subsets. Different clinical issues are pressing for the different types of breast cancers, better systemic therapies are needed for triple receptor-negative cancers, proper sequencing and length of adjuvant endocrine therapy is an issue yet to be clarified for endocrine-sensitive ER-positive cancers, and optimal use of chemotherapy and better drugs are needed for the poor-prognosis ER-positive cancers. It is also apparent that different biomarkers are needed for the different cancer subsets. For example, proliferation-related markers are strongly prognostic and also predictive of chemotherapy sensitivity among ER-positive cancers but these same markers are not predictive in ER-negative cancers.

Traditional breast cancer studies where all patients with cancer of the breast are eligible for the same therapy will soon be regarded as naïve 
as a clinical trial proposal to treat all acute and chronic leukemias with the same drug in a single trial and perform subset analysis for the various cytologic types at the end.

\section{S5}

\section{Clinical utility and future of genetic profiles for breast cancer \\ C Hudis ${ }^{1,2}$}

${ }^{1}$ Memorial Sloan-Kettering Cancer Center, New York, NY, USA; ${ }^{2}$ Weill Cornell Medical College, New York, NY, USA

Breast Cancer Research 2009, 11(Suppl 1):S5 (doi: 10.1186/bcr2266)

The era of breast cancer as a single disease, and one-size-fits-all treatment, has passed. Hormone receptor status has allowed us to identify two phenotypic subtypes of breast cancer superimposed on the existing light microscopic histologic classifications, and in recent years HER2 has added a third axis of categorization. These three markers (ER, PR, and HER2) share the fact that they are linked inextricably to treatment decisions as the functional targets of specific agents and therefore to a degree the treatment has defined the disease. With the availability of molecular subtyping relying on mRNA in paraffin-embedded tissue or fresh-frozen material, precise assessments of gene loci for amplification, deletion, or mutation, and the development of high-throughput techniques, we now are at the beginning of an era when it may be possible and appropriate to generate a genetic profile for each patient's tumor such that we will subset breast cancer further and will tailor therapy for these subsets. Already two commercial tests are available to clinicians (Mammaprint and OncytopeDx), each offering prognostic information based on a collection of genes - and the latter also providing predictive information with regard to the value of chemotherapy. Each is currently being tested in prospective studies to provide additional information about how best to integrate them into routine care. On the horizon are newer and potentially more informative techniques, such as representational oligonucleotide microarray, a version of comparative genomic hybridization, which can provide more detail regarding gene amplifications and deletions. This presentation will review the available technologies and discuss their potential clinical utility.

\section{SYMPOSIUM II}

\section{New surgical approaches}

\section{S6}

\section{Axillary staging: new approaches and treatment of minimal disease MTFD Vrancken Peeters}

Department of Surgery, National Cancer Institute, Antoni van Leeuwenhoek Hospital, Amsterdam, the Netherlands

Breast Cancer Research 2009, 11(Suppl 1):S6 (doi: 10.1186/bcr2267)

Axillary staging is necessary since 30 to $40 \%$ of patients with curable breast cancer will have metastases in the axilla. Years ago, the most radical method of staging was performed: an axillary clearance. However, in patients with a negative axilla, who make up 60 to $70 \%$ of all patients, this lymph node dissection has no additional value. Today, with the sentinel node procedure it is possible to offer patients accurate staging of the axilla without the morbidity of axillary clearance. Discussion has now started about the fact that there are subgroups of patients in which the risk of axillary metastases is so low that axillary staging can be omitted. Furthermore, it is standard practice to perform axillary clearance in patients with positive sentinel lymph nodes. However, studies show that, of patients with a positive sentinel lymph node, 40 to $60 \%$ have no additional involved lymph nodes.

The following dilemmas, which are all related to minimal disease, are therefore still open for debate and will be discussed in the presentation: Is a sentinel node procedure necessary when only minimal disease is present in the breast? Is axillary treatment necessary when only minimal disease is present in the node? What is the role of the PET/CT scan in axillary staging in minimal disease? How can we stage the axilla after primary systemic treatment when potentially only still minimal disease is present?

\section{S7}

\section{Surgical management of the breast and axilla after neoadjuvant treatment: the role of sentinel node biopsy} EP Mamounas ${ }^{1,2}$

${ }^{1}$ Aultman Cancer Center, Canton, OH, USA; ${ }^{2}$ Northeastern Ohio Universities College of Medicine, Rootstown, OH, USA

Breast Cancer Research 2009, 11(Suppl 1):S7 (doi: 10.1186/bcr2268)

Several unique surgical issues arise in the management of patients who are selected to receive neoadjuvant systemic therapy. These involve the original diagnostic assessment of the extent of disease in the breast and axilla, the preoperative planning, and ultimately the surgical management of the primary breast tumor and that of axillary lymph nodes. Careful consideration of these issues is critical in order to maximize local control of the disease, while minimizing the extent of the required surgical resection and the ensuing surgical morbidity.

Adequate diagnostic assessment with core needle biopsy before initiation of neoadjuvant systemic therapy ensures the presence of invasive carcinoma and provides adequate material for routine biomarker evaluation (such as ER, PR and HER-2 neu), while minimally disturbing the primary breast tumor. Consideration should also be given to assessing the status of axillary nodes by minimally invasive techniques such as ultrasound of the axilla with fine needle aspiration of suspicious nodes. Optimal preoperative planning aims at accurately determining the patterns of primary tumor shrinkage and the amount and location of any residual disease in the breast.

Surgical treatment after neoadjuvant systemic therapy focuses on the management of the primary breast tumor and that of axillary lymph nodes. Regarding the primary breast tumor, several studies have shown that neoadjuvant systemic therapy converts a proportion of mastectomy candidates to candidates for breast-conserving surgery. Neoadjuvant systemic therapy can also decrease the amount of breast tissue that needs to be removed at lumpectomy even in patients who are lumpectomy candidates at presentation.

Neoadjuvant systemic therapy (primarily neoadjuvant chemotherapy) downstages axillary lymph nodes in up to 30 to $40 \%$ of the patients. Although this observation was of little clinical significance when axillary node dissection was the sole method for staging the axilla, the development and validation of sentinel lymph node biopsy (SNB) has provided an additional potential advantage for neoadjuvant chemotherapy; that is, the possibility of decreasing the extent and morbidity of axillary surgery. This approach is, naturally, predicated on the premise that SNB is feasible and accurate following neoadjuvant chemotherapy. Initially, small, single-institution studies examined the efficacy of lymphatic mapping and the accuracy of SNB after neoadjuvant chemotherapy with significant variability in the rate of SN identification and in the rate of false negative SN [1]. However, when these studies are examined collectively $[1,2]$ or when larger, multicenter datasets are analyzed [3], SNB after neoadjuvant chemotherapy appears to have similar performance characteristics to those of SNB before systemic therapy [4-6].

Some have proposed that candidates for neoadjuvant systemic therapy should have SNB before, rather than after, neoadjuvant systemic therapy so that information on the status of the axillary nodes be obtained without the potential confounding effects of systemic treatment, and sentinel node-negative patients can avoid axillary dissection [7-9]. Although this approach may be useful in patients who will not need systemic therapy (that is, chemotherapy) if the SN is negative, it is not generally useful for the majority of candidates for neoadjuvant systemic therapy, for whom little - if any - is to be gained by knowing the pathologic nodal status upfront. In addition, this approach commits patients to two surgical procedures and does not 
take advantage of the downstaging effect of neoadjuvant chemotherapy on the axillary nodes.

References

1. Mamounas EP: Sentinel lymph node biopsy after neoadjuvant systemic therapy. Surg Clin North Am 2003, 83:931-942.

2. Xing $Y$, Ding D, Cox D, et al.: Meta-analysis of sentinel lymph node biopsy following preoperative chemotherapy in patients with operable breast cancer [abstract 561]. Proc Am Soc Clin Oncol 2004, 23:17.

3. Mamounas EP, Brown A, Anderson S, et al:: Sentinel node biopsy after neoadjuvant chemotherapy in breast cancer: results from National Surgical Adjuvant Breast and Bowel Project Protocol B-27. J Clin Oncol 2005, 23:2694-2702.

4. Veronesi U, Paganelli G, Viale G, et al:: A randomized comparison of sentinel-node biopsy with routine axillary dissection in breast cancer. N Engl J Med 2003, 349:546-553.

5. McMasters KM, Tuttle TM, Carlson DJ, et al:: Sentinel lymph node biopsy for breast cancer: a suitable alternative to routine axillary dissection in multi-institutional practice when optimal technique is used. J Clin Onco/ 2000, 18:2560-2566.

6. Krag D, Weaver D, Ashikaga T, et al.: The sentinel node in breast cancer - a multicenter validation study. N Engl J Med 1998, 339:941-946.

7. Bedrosian I, Reynolds C, Mick R, et al.: Accuracy of sentinel lymph node biopsy in patients with large primary breast tumors. Cancer 2000, 88:2540-2545.

8. Schrenk P, Hochreiner G, Fridrik M, et al.: Sentinel node biopsy performed before preoperative chemotherapy for axillary lymph node staging in breast cancer. Breast $J$ 2003, 9:282-287.

9. Sabel MS, Schott AF, Kleer CG, et al.: Sentinel node biopsy prior to neoadjuvant chemotherapy. Am J Surg 2003, 186:102-105.

\section{S8}

\section{Immediate versus delayed repair of partial} mastectomy defects in breast conservation SJ Kronowitz, KK Hunt, H Kuerer, E Strom, TA Buchholz, JE Ensor, CA Koutz, GL Robb

The University of Texas MD Anderson Cancer Center, Houston, TX, USA Breast Cancer Research 2009, 11(Suppl 1):S8 (doi: 10.1186/bcr2269)

Introduction The authors previously compared the local tissue rearrangement, breast reduction, and latissimus dorsi flap reconstruction techniques for repairing partial mastectomy defects and showed the benefits of breast reduction.

Methods In the present study, the authors focused solely on factors influencing outcome in 41 patients who underwent repair of a partial mastectomy defect using breast reduction.

Results Tumor location had a significant effect on the design of the parenchymal pedicle $(P=0.05)$. Most repairs were performed with an inferior pedicle. Fifty percent of the lower outer and central quadrant tumors required an amputative design with a free nipple graft. The complication rates for immediate and delayed repair were $24 \%$ and $50 \%$, respectively. The superior pedicle was associated with the highest complication rates. Tumors in the upper outer quadrant of the breast were associated with the highest complication rate (35\%). Ninety percent of patients with planned repairs had a viable nipple-areola complex $(P=0.05)$ and did not require a free nipple graft. More favorable cosmetic outcomes were achieved using an inferior pedicle; less favorable cosmetic outcomes were achieved for tumors in the upper inner quadrant of the breast. Larger defects did not result in less favorable cosmetic outcomes than smaller defects. Only $7 \%$ of patients had a positive tumor margin. Five percent of patients developed local breast cancer recurrence after a mean follow-up of 36 months.

Conclusions The authors provide practical guidelines for repairing a partial mastectomy defect using breast reduction that should minimize the occurrence of complications and optimize the cosmetic outcome [1]. Reference reduction technique in patients undergoing breast conservation therapy. Plast Reconstr Surg 2007, 120:1755-1768.

\section{S9}

\section{Prophylactic contralateral surgery: current recommendations and techniques M Morrow}

Memorial Sloan-Kettering Cancer Center, New York, NY, USA

Breast Cancer Research 2009, 11(Suppl 1):S9 (doi: 10.1186/bcr2270)

The use of contralateral prophylactic mastectomy (CPM) from both invasive cancer and ductal carcinoma in situ is increasing in the United States [1], in spite of the fact that only $3 \%$ of breast cancer patients in the Surveillance, Epidemiology, and End Results database developed a contralateral cancer at 5 years [2]. Endocrine therapy and trastuzumab, both widely used as adjuvant therapy, reduce the incidence of contralateral cancer by about $50 \%$, suggesting that the risk of contralateral cancer in women treated today is quite low.

Most guidelines for prophylactic mastectomy address women at high risk, not women with unilateral cancer. Known or suspected BRCA mutation carriers who develop unilateral cancer have a 40 to $60 \%$ risk of developing a contralateral cancer, and CPM is accepted as appropriate therapy in this circumstance.

To address the issue of what kind of women undergo CPM, we identified 477 patients who had the procedure at Memorial SloanKettering Cancer Center within 1 year of a unilateral cancer diagnosis and compared them with 2,500 women undergoing unilateral mastectomy in the same period (1997 to 2005). CPM patients were younger (median 46 vs 53 years; $P<0.0001$ ), had smaller tumors (1.2 vs 1.8 $\mathrm{cm} ; P<0.0001$ ), and were less likely to have positive nodes (47 vs 57\%; $P<0.0001$ ). Hormone receptor and HER2 status did not differ between cases and controls. Although $68 \%$ of CPM patients reported a family history of breast cancer, only $2 \%$ underwent genetic testing. Of these, $34(7 \%)$ were found to have a mutation. The pathology specimens of $6 \%$ of patients undergoing CPM were found to contain malignancy (eight invasive, 20 ductal carcinoma in situ).

When CPM is performed, it should be done to the same anatomic limit as a therapeutic mastectomy. Skin sparing to facilitate reconstruction is appropriate, but flaps should be the same thickness as is used in a therapeutic mastectomy. The use of nipple sparing is controversial [3]. In order to maintain a blood supply to the nipple areolar complex, some breast tissue must be left behind. Most studies of local recurrence after nipple preservation have been limited to patients with breast cancer, and little is known about its use in the prophylactic setting, particularly regarding risks in BRCA carriers. However, local recurrence is uncommon after nipple sparing for cancer treatment, and improved body image and psychological adjustment after nipple sparing has been reported [3], making this option worthy of consideration in patients undergoing CPM.

References

1. Tuttle TM, Jarosek S, Habermann EB, Arrington A, Abraham A, Morris TJ, Virnig BA: Increasing rates of contralateral prophylactic mastectomy among patients with ductal carcinoma in situ. J Clin Oncol 2009, 27:1362-1367.

2. Gao X, Fisher SG, Emami B: Risk of second primary cancer in the contralateral breast in women treated for early-stage breast cancer: a population-based study. Int J Radiat Oncol Biol Phys 2003, 56:1038-1045.

3. Chung A, Sacchini V: Nipple-sparing mastectomy: where are we now? Surg Oncol 2008, 17:261-266. 


\section{SYMPOSIUM III}

\section{Advances in radiotherapy}

\section{S10}

\section{Other new radiotherapy techniques SC Formenti}

New York University School of Medicine, New York, NY, USA

Breast Cancer Research 2009, 11(Suppl 1):S10 (doi: 10.1186/bcr2271)

Objective To report the NYU research on novel radiation therapy of breast cancer. Radiation therapy has enabled effective breast preservation in the majority of newly diagnosed breast cancer patients. This milestone in the history of breast cancer management is currently revisited to identify the optimal selection of target and fractionation, while assuring minimal radiation exposure of normal tissues adjacent to the breast.

Methods Five consecutive prospective trials explored hypofractionated, accelerated regimens of breast radiotherapy that also aim at optimal normal tissue sparing.

Results After pilot-testing a 3-week prone regimen of IMRT to the breast with a concomitant boost to the tumor bed, we have prospectively studied in a cohort of 400 women whether a prone versus a supine setup for treatment was superior at sparing lung and heart tissue, while assuring target (index breast) coverage. The results of this trial indicate that the prone setup is superior in $>90 \%$ of patients.

The prone setup also characterizes our two studies of partial breast irradiation delivered over five fractions of 6 Gy each. At NYU this approach is only offered to the subset of patients at the lowest risk of local recurrence after breast-conserving surgery, postmenopausal women with $\mathrm{T} 1$ lesions, resected with negative margins. Results at a median follow-up of 5 years demonstrate $2 \%$ local recurrence rate.

Conclusions Breast radiotherapy after breast-conserving surgery can be safely delivered over 3 weeks. A prone technique enables optimal sparing of the lung and heart in the majority of patients. Current research focuses on a prone setup that includes level III and supraclavicular lymph nodes in patients with positive lymph nodes, to enhance sparing of the lung and heart.

\section{SYMPOSIUM IV}

\section{Changes in preoperative treatment}

\section{S11}

\section{Biomarkers and predictive factors of response to neoadjuvant treatment}

M Dowsett' ${ }^{1}$, A Dunbier ${ }^{1}$, H Anderson'1, J Salter', S Detre', R Jones ${ }^{1}$, A Skene ${ }^{2}$, M Dixon ${ }^{3}$, IE Smith'

${ }^{1}$ Royal Marsden Hospital, London, UK; ${ }^{2}$ Royal Bournemouth Hospital, Bournemouth, UK; ${ }^{3}$ Western General Hospital, Edinburgh, UK

Breast Cancer Research 2009, 11(Suppl 1):S11 (doi: 10.1186/bcr2272)

Introduction Neoadjuvant therapy provides a unique and powerful opportunity to derive biopsy material before, during and subsequent to the treatment of otherwise untreated breast cancers and, by measuring the expression of biomarkers in these, to study the biology of the disease in vivo. We have conducted such studies involving endocrine therapy or chemotherapy and have focused on the concept that measurement of change in expression of critical biomarkers shortly after starting therapy may be more closely associated with clinical outcome than before therapy.

Endocrine therapy The IMPACT trial, in which over $300 \mathrm{ER}^{+}$patients were treated with tamoxifen or anastrozole or the combination, revealed that change in the proliferation marker Ki67 was greater with anastrozole than with either of the other arms, a result parallel to the drug's greater effectiveness in the far larger and longer ATAC adjuvant trial. In addition, Ki67 after 2 weeks was more predictive of recurrencefree survival than pretreatment Ki67 [1]. The value of on-treatment Ki67 as an index of long-term outcome is being studied in detail in the 4,000-patient Perioperative Endocrine Treatment for Individualised Care (POETIC) trial. The combination of on-treatment Ki67 with standard clinical features has allowed the derivation of a Preoperative Endocrine Therapy Index, which identified a group of patients with a very low likelihood of relapse on endocrine treatment alone [2]. Most recently we have created expression array data from over 100 patients treated with anastrozole. The quantitative expression of ER at a transcript level correlated strongly with the decrease in Ki67 and a Global Index of Dependence on Estrogen such that tumours with low ER showed little reaction to oestrogen deprivation. However, not all tumours with high ER expression showed high oestrogen dependence. Some of the less dependent tumours were HER2-positive but other mechanisms must account for the discordance in others. The POETIC trial should help to identify molecular factors associated with resistance to aromatase inhibitors.

Conclusions Neoadjuvant chemotherapy is also associated with reduced levels of Ki67, although the mechanism in this case is likely to involve the selective apoptosis of highly proliferative cells as opposed to the cytostatic effect of endocrine therapy. In general those molecular features that are associated with poor long-term outcome are related to good response to neoadjuvant chemotherapy, and this complicates the use of the presurgical setting for study of treatment efficacy. Patients with high proliferation and ER negativity are more likely to show pathological complete response; the higher proliferation seen in ERnegative tumours appears to only partly explain the greater effectiveness seen in these cases. Those tumours with high Ki67 at the end of neoadjuvant therapy have a very poor outcome [3].

References

1. Dowsett M, Smith IE, Ebbs SR, Dixon JM, Skene A, A'Hern R, Salter J, Detre S, Hills M, Walsh G, IMPACT Trialists Group: Prognostic value of Ki67 expression after short-term presurgical endocrine therapy for primary breast cancer. J Natl Cancer Inst 2007, 99:167-170.

2. Ellis MJ, Tao Y, Luo J, A'Hern R, Evans DB, Bhatnagar AS, Chaudri Ross HA, von Kameke A, Miller WR, Smith I, Eiermann W, Dowsett M: Outcome prediction for estrogen receptor-positive breast cancer based on postneoadjuvant endocrine therapy tumor characteristics. J Natl Cancer Inst 2008, 100:1380-1388.

3. Jones et al:: The prognostic significance of Ki67 before and after neoadjuvant chemotherapy in breast cancer. Breast Cancer Res Treat 2008. [Epub ahead of print]

\section{S12}

\section{Adjuvant therapy after preoperative treatment for breast cancer \\ E Ciruelos}

Hospital Universitario 12 de Octubre, Madrid, Spain

Breast Cancer Research 2009, 11(Suppl 1):S12 (doi: 10.1186/bcr2273)

The increase in the use of preoperative chemotherapy has raised new questions regarding how to optimize locoregional and systemic adjuvant treatment. When patients are given preoperative systemic therapy, the preferred therapeutic regimens are the same as those established as safe and active in the adjuvant setting. At present, no data suggest that systemic treatment should be tailored, in one direction or another, based on initial tumor response or lack thereof (except for frank disease progression while on treatment), or based on the extent of residual disease.

Adjuvant locoregional and systemic treatment after preoperative chemotherapy for breast cancer is still controversial. No high-quality data from prospective trials are available; nevertheless, locoregional therapy decisions should be based on both pretreatment and the clinical extent of disease. Sentinel node biopsy can be performed before and after preoperative therapy in patients with clinical NO 
disease at diagnosis. It is not clear whether resection margins should be differently evaluated after preoperative therapy than in the standard setting. The success of breast-conserving surgery depends on careful patient selection and on an adequate surgical technique that achieves negative margins. Adjuvant breast irradiation is indicated for all patients who undergo breast conservation; for patients treated with mastectomy, chest-wall and regional nodal radiation must be done in those who present with clinical stage III disease or who have histologically positive lymph nodes at diagnosis.

Additional studies are needed to determine the value of postmastectomy irradiation in clinical stage II breast cancer, as well as to determine the convenience of adjuvant systemic therapy in patients who do not achieve a good pathological response with preoperative treatment. Multidisciplinary treatment teams are critical in order to improve therapeutic management of these patients.

\section{SYMPOSIUM V}

\section{Advances in adjuvant therapy}

\section{S13}

\section{Adjuvant therapy of HER2 ${ }^{+}$breast cancer EP Winer}

Dana-Farber Cancer Institute, Boston, MA, USA

Breast Cancer Research 2009, 11(Suppl 1):S13 (doi: 10.1186/bcr2274)

Adjuvant therapy of HER2 ${ }^{+}$breast cancer has evolved dramatically over the past 4 years. It is now widely appreciated that treatment with trastuzumab, when administered concurrently or sequentially with chemotherapy, will decrease the risk of disease recurrence by approximately $50 \%$. Clinical trials have also demonstrated a modest improvement in overall survival, and it is anticipated that this survival advantage will increase with further follow-up. Nevertheless, there are patients who will experience disease recurrence in spite of optimal trastuzumab-based therapy, and new treatment approaches are needed for these individuals. The results of the initial randomized trials have also given rise to a number of difficult questions that clinicians and patients face on a daily basis. The presentation will focus on controversial topics in the adjuvant treatment of $\mathrm{HER}^{+}$disease, including: the treatment of small, node-negative tumors; the uncertainty surrounding intermediate HER2 ${ }^{+}$test results; and the concerns about cardiac toxicity. New treatment approaches for HER2+ breast cancer that hold promise for the adjuvant setting will also be discussed.

\section{S14}

\section{Controversies in hormonal adjuvant therapy for premenopausal patients}

\section{A Howell}

The Christie NHS Foundation Trust, Paterson Institute for Cancer

Research, Manchester, UK

Breast Cancer Research 2009, 11(Suppl 1):S14 (doi: 10.1186/bcr2275)

The major controversy concerning adjuvant hormonal therapy for premenopausal patients is the absence of appropriate clinical trial data to indicate the appropriate way to treat patients presenting with oestrogen receptor (ER)-positive operable breast cancer. This is despite the fact that the first randomized trial in breast cancer, begun in 1947, was a comparison of ovarian irradiation versus no further treatment after primary surgery for breast cancer [1] and the availability of recent overviews $[2,3]$. The Oxford overview indicates that tamoxifen is as effective in premenopausal women as in postmenopausal women. It also indicates that ovarian ablation is effective alone but not in addition to chemotherapy [3]. Subsequent studies indicate that ovarian suppression is effective in addition to chemotherapy in young women who do not develop chemotherapy-induced amenorrhoea. Multiple randomised studies indicate in patients with ER-positive tumours that ovarian suppression with or without tamoxifen is as effective as chemotherapy. All of these studies have been of poor design since none has had a third arm where both endocrine therapy and chemotherapy are given. It is likely that in the chemotherapy-alone arm there would be additional patients who would have responded to endocrine therapy and vice versa so that, although no differences between endocrine and chemotherapy were seen in these trials, it is not logical to conclude that either treatment alone is optimal without appropriate trial data. Because trials comparing methods of ovarian suppression have relatively few patients, we do not know the most effective method. LHRH agonists are associated with temporary ovarian suppression. The ZEBRA trial demonstrated that 2 years of goserelin was as effective as chemotherapy, but in this and subsequent trials of the use of LHRH agonists we do not have a clear indication of the duration of treatment required. Finally, no adequate trials indicate whether an LHRH agonist adds to the effectiveness of tamoxifen as adjuvant therapy. Given the uncertainties of treatment of premenopausal ER-positive early breast cancer, it is vital that patients are entered into appropriate trials such as SOFT.

References

1. Paterson R, Russell MH: Clinical trials in malignant disease. Part II - breast cancer, value of irradiation of the ovaries. J Fac Radiol 1959, 10:130-133.

2. LHRH-agonists in Early Breast Cancer Overview Group; Cuzick J, Ambroisine L, Davidson N, Jakesz R, Kaufmann M, Regan M, Sainsbury R: Use of luteinising-hormone-releasing hormone agonists as adjuvant treatment in premenopausal patients with hormone-receptor-positive breast cancer: a meta-analysis of individual patient data from randomised adjuvant trials. Lancet 2007, 3691:711-723.

3. Early Breast Cancer Trialists' Collaborative Group: Chemotherapy and hormonal therapy for early breast cancer: effects on recurrence and 15 year survival in an overview of the randomised trials. Lancet 2005, 365:1687-1717.

\section{S15}

\section{Controversies in the hormonal adjuvant therapy of postmenopausal patients \\ M Dowsett}

Royal Marsden Hospital, London, UK

Breast Cancer Research 2009, 11(Suppl 1):S15 (doi: 10.1186/bcr2276)

Over $80 \%$ of primary breast cancers in postmenopausal women present as $\mathrm{ER}^{+}$. It is clear that aromatase inhibitors (Als) are more effective than tamoxifen as initial adjuvant therapy for such patients. Overview analysis indicates that this proportional enhancement in benefit is seen across all common clinical subgroups [1]. Recent data from the BIG1-98 trial [2] revealed that initial therapy with tamoxifen with a switch after 2 years to letrozole was not as effective as continued letrozole, an important refutation of earlier modelling that suggested the sequential approach might be at least as effective as 5 years' Al treatment. The indication that 2 years of letrozole followed by 3 years of tamoxifen therapy is as effective as 5 years of letrozole provokes new thinking about the possible best use of these agents.

Tamoxifen has some serious side effects (for example, increased risk of endometrial cancer and thromboembolism) that Als do not, but the deleterious bone and joint effects of Als make tamoxifen a continued choice of treatment in some patients, particularly those at low risk of relapse.

The early indications that Als might be relatively more effective than tamoxifen in $\mathrm{PgR}^{-}$versus $\mathrm{PgR}^{+}$patients and $\mathrm{HER}^{+}$versus HER2tumours have not been confirmed. Similarly, the Oncotype DX Recurrence Score (RS) showed similar relationships with risk of distant recurrence in both anastrozole and tamoxifen arms of the ATAC trial. Although data from BIG1-98 show a greater effect of Als over tamoxifen at higher levels of Ki67, there is no significant interaction between the Ki67 and treatment effects - suggesting that this is largely an effect of high Ki67 being associated with poorer prognosis. There are conflicting data on whether polymorphisms in the CYP2D6 gene, which reduce the efficiency of conversion of tamoxifen to the 
more potent endoxifen, indicate a poorer efficacy for tamoxifen and therefore greater relative benefit from an Al.

The decision to deliver adjuvant chemotherapy in addition to endocrine treatment is judged largely on the basis of prognosis after considering the impact of the endocrine therapy. While the RS is a validated instrument for this purpose, the prognostic effect of markers such as $\mathrm{PgR}, \mathrm{HER} 2$ and Ki67 is substantial and may allow the development of widely applicable immunohistochemical approaches to risk evaluation. References

1. Ingle et al.: [Abstract 12]. Cancer Res 2009, 69(Suppl):66s.

2. Mouridsen et al.: [Abstract 13]. Cancer Res 2009, 69(Suppl): $66 s$.

\section{SYMPOSIUM VII}

\section{Bone-targeted therapies}

\section{S16}

\section{Targeting the tumour microenvironment: denosumab, a new RANKL inhibitor \\ RE Coleman}

Academic Unit of Clinical Oncology, Weston Park Hospital, Sheffield, UK

Breast Cancer Research 2009, 11(Suppl 1):S16 (doi: 10.1186/bcr2277)

Bone is the most common site for metastasis and is of particular clinical importance in breast cancer, which is common and associated with a relatively long clinical course. Metastatic bone disease results from the interactions between cancer cells in the bone marrow microenvironment and normal bone cells rather than direct destruction by cancer cells. These growth factor and cytokine-mediated interactions typically lead to stimulation of both osteoclast and osteoblast function with uncoupling and imbalance in bone remodelling. This provides the rationale for bone-targeted therapies to reduce the risk of skeletal complications such as fracture, and to relieve bone pain. Additionally, bone-derived growth factors released from bone promote a fertile environment for the survival and proliferation of cancer cells, creating a vicious cycle of bone destruction. Receptor activator of NF- $K B$ ligand (RANKL) is a key mediator in this process. Within the bone microenvironment, factors secreted by tumour cells stimulate stromal cells and osteoblasts to secrete RANKL, which binds to its cognate receptor RANK on the surface of precursor and mature osteoclasts. RANKL is a critical mediator of osteoclast differentiation, function, and survival.

Denosumab is a fully humanised monoclonal antibody that inhibits RANKL. A dose of $120 \mathrm{mg}$, 4-weekly, administered by subcutaneous injection has been defined in a large dose-finding randomised phase II study for the treatment of advanced malignancy [1]. To prevent treatment-induced bone loss, an osteoporosis dose and schedule of $60 \mathrm{mg}$ every 6 months has been evaluated, and was shown to prevent aromatase inhibitor-induced bone loss [2]. The large phase III trials in metastatic bone disease comparing denosumab with zoledronic acid have completed accrual and will report in the next year.

Preclinical data suggest that denosumab is a more complete inhibitor of osteoclast function than the bisphosphonates (BPs). Additionally, a randomised phase II study in patients with increased bone resorption despite ongoing $\mathrm{BPs}$ has compared changing to denosumab, an antibody to RANK ligand, with continuation of the BP. This showed rapid and sustained biochemical response in $>80 \%$ of patients with denosumab compared with $<30 \%$ for those on standard BP treatment. Additionally, the number of skeletal events appeared to be less in the denosumab-treated patients [3]. The Austrian Breast Cancer Study Group are conducting a large randomised trial in postmenopausal women receiving endocrine treatment with the $60 \mathrm{mg}$ every 6 months schedule. This is primarily to evaluate effects on bone mineral density and fractures, but with disease-free survival as a secondary endpoint. Other metastasis prevention trials are planned.

\section{References}

1. Lipton A, Steger GG, Figueroa J, et al.: Extended efficacy and safety of denosumab in breast cancer patients with bone metastases not receiving prior bisphosphonate therapy. Clin Cancer Res 2008, 14:6690-6696.

2. Ellis GK, Bone HG, Chlebowski R, et al:: Effect of denosumab on bone mineral density in women receiving adjuvant aromatase inhibitors for non-metastatic breast cancer: subgroup analyses of a phase 3 study. Breast Cancer Res Treat 2009. [Epub ahead of print]

3. Fizazi K, Lipton A, Mariette X, et al.: Randomized phase II trial of denosumab in patients with bone metastases from prostate cancer, breast cancer, or other neoplasms after intravenous bisphosphonates. J Clin Oncol 2009. [Epub ahead of print]

\section{S17}

\section{Bisphosphonates in the adjuvant treatment of early breast cancer M Gnant}

Department of Surgery, Medical University of Vienna, Austria Breast Cancer Research 2009, 11(Suppl 1):S17 (doi: 10.1186/bcr2278) Bisphosphonates are the standard of care for preventing skeletal morbidity and treating hypercalcemia of malignancy in patients with bone metastases. Zoledronic acid (intravenous; $4 \mathrm{mg}$ monthly) is approved to prevent skeletal-related events in patients with bone metastases from several tumor types, and can improve survival in some subsets of patients with skeletal metastases and high baseline bone turnover. In the adjuvant setting, bisphosphonates have shown clinical efficacy for preventing cancer treatment-induced bone loss and promise for reducing disease recurrence. For example, early studies of clodronate showed the potential for bisphosphonates to prevent bone metastases and prolong survival, but results with clodronate have been inconsistent. Recently, the more active bisphosphonate zoledronic acid (4 mg every 6 months) prevented bone loss and significantly reduced the risk of disease-free survival events by $36 \%(P=0.01)$ compared with adjuvant endocrine therapy alone in a large phase III trial $(n=1,803)$ in premenopausal women with early breast cancer [1]. Notably, these benefits were not limited to bone because the addition of zoledronic acid reduced disease recurrence at all sites. This fuels the See-and-Soil hypothesis about dormant tumor (stem) cells in early disease, and hints towards a potential impact of bisphosphonate treatment on the bone marrow microenvironment. These results of twice-yearly zoledronic acid have been confirmed indirectly in boneprotection trials in postmenopausal patients [2]. In addition, several ongoing trials (involving more than 20,000 patients altogether) are evaluating the efficacy of bisphosphonates for the prevention of metastases in breast, prostate, and lung cancers, and multiple myeloma. Results from these studies are likely to expand the role of bisphosphonates, particularly zoledronic acid, in the adjuvant therapy setting, and help us in elucidating the underlying biology as well as resolving open clinical questions.

Acknowledgements The authors acknowledge the invaluable contribution of our patients who contributed to ABCSG-12 and other ABCSG trials as well as the work of all ABCSG investigators, study nurses, and data-management associates, both in the individual trial centers and in the ABCSG center. ABCSG-12 is an academic trial that received support from AstraZeneca and Novartis.

References

1. Gnant M, Mlineritsch B, Schippinger W, et al.: Endocrine therapy plus zoledronic acid in premenopausal breast cancer. $N$ Engl $J$ Med 2009, 360:679-691.

2. Eidtmann H, Bundred N, De Boer R, et al:: The effect of zoledronic acid on aromatase inhibitor associated bone loss in postmenopausal women with early breast cancer receiving letrozole: $\mathbf{3 6}$ months follow-up of ZO-FAST [abstract 44]. Presented at 31st San Antonio Breast Cancer Symposium; 10-14 December 2008; San Antonio, TX. 


\section{S18}

\section{Breast tumor heterogeneity: causes and consequences}

\section{K Polyak, M Shipitsin, LL Campbell-Marrotta, N Bloushtain-} Qimron, So Yeon Park

Department of Medical Oncology, Dana-Farber Cancer Institute, Harvard Medical School, Boston, MA, USA

Breast Cancer Research 2009, 11(Suppl 1):S18 (doi: 10.1186/bcr2279)

Introduction In breast and other cancer types, a high degree of diversity exists among and within tumors. Breast tumors are composed of a variety of cancer cells with distinct phenotypes and genotypes. The molecular mechanisms responsible for this intratumoral heterogeneity are not well defined. Two currently popular hypotheses that attempt to explain intratumoral heterogeneity are the cancer stem cell and the clonal evolution models. Each of these concepts has been investigated for some time, leading to the accumulation of findings that support one or the other. Although the two views share some similarities, they are fundamentally different notions with very different implications for clinical practice.

Methods To characterize cells with stem-like characteristics, we determined the gene expression, DNA methylation, and genetic profiles of distinct cell populations purified from breast carcinomas and normal breast tissue using cell surface markers CD24 and CD44 that have been associated with stem cell-like properties. Gene expression profiles were analyzed using serial analysis of gene expression, DNA methylation profiles by methylation-specific digital karyotyping, whereas genetic alterations were investigated using SNP arrays and fluorescence in situ hybridization.

Results SNP array and methylation-specific digital karyotyping analyses suggested that CD24+/CD44- and CD24-/CD44+ cells from the same tumor are clonally related, but can be both genetically and epigenetically distinct. CD $44^{+}$cells have an activated TGF $\beta$ signaling pathway, whereas it is decreased in $\mathrm{CD}_{24}{ }^{+}$cells due to TGF $\beta R 2$ promoter hypermethylation. As a consequence of this, $\mathrm{CD} 44^{+}$cells specifically respond to an inhibitor of TGF $\beta R$, and acquire more differentiated epithelial cellular morphology and membrane localization of $\mathrm{E}$-cadherin and $\beta$-catenin. Furthermore, gene expression profiling revealed that breast cancer patients with lymph node-negative breast tumors that have a higher fraction of $\mathrm{CD} 44^{+}$cells had shorter overall survival as well as shorter distant metastasis-free survival. In contrast, CD24 ${ }^{+}$cells appeared to be more prevalent in distant metastases even when the primary breast tumor was enriched for CD44+ cells. This suggests that the tumor cells may be altered during the metastatic process, or that $\mathrm{CD} 24^{+}$breast cancer cells are intrinsically more competent for metastasis.

Conclusions The results of our recent studies investigating breast cancer cells with stem cell characteristics and the genetic diversity of cellular populations within tumors imply multiple layers of heterogeneity and a combination of different molecular mechanisms underlying breast tumor heterogeneity. Understanding these molecular mechanisms will facilitate the development of more effective ways to treat and prevent breast cancer.

References

1. Shipitsin M, Campbell LL, Argani P, Weremowicz S, Noga Bloushtain-Qimron N, Yao J, Nikolskaya T, Serebryiskaya T, Beroukhim R, Hu M, et al.: Molecular definition of breast tumor heterogeneity. Cancer Cell 2007, 11:259-273.

2. Polyak K, Weinberg RA: Transitions between epithelial and mesenchymal states: acquisition of malignant and stem cell features. Nat Rev Cancer 2009, 9:265-273.

3. Bloushtain-Qimron N, Yao J, Snyder EL, Shipitsin M, Campbell LL, Mani SA, Hu M, Chen H, Ustyansky V, Antosiewicz JE, et al:: Celltype specific DNA methylation patterns in the human breast. Proc Natl Acad Sci 105:14076-14081.

4. Campbell LL, Polyak K: Breast tumor heterogeneity: cancer stem cells or clonal evolution? Cell Cycle 2007; 6:2332-2338.

\section{SYMPOSIUM VIII}

\section{New drugs beyond herceptin}

\section{S19 \\ Neratinib, an irreversible pan erB receptor tyrosine kinase inhibitor active for advanced HER2 ${ }^{+}$breast cancer \\ H Cortés-Funes, C Mendiola, L Manso, E Ciruelos \\ Servicio Oncología Médica, Hospital Universitario 12 de Octubre, Madrid, Spain}

Breast Cancer Research 2009, 11(Suppl 1):S19 (doi: 10.1186/bcr2280)

Neratinib (HKI-272) is a dual inhibitor of the tyrosine kinase receptors, erbB1 (EGFR) and erbB2 (HER2). In a phase I study, neratinib was tolerable and demonstrated antitumor activity in patients with solid tumors, including 8 of 25 evaluable patients with erbB2-positive advanced breast cancer. In an open-label, two-arm phase II study, patients with stage IIIB, IIIC or IV erbB2-positive advanced breast cancer were evaluated to further characterize the safety and efficacy of neratinib. The maximum tolerated dose of once-daily oral neratinib is $320 \mathrm{mg}$. The most common neratinib-related toxicity was diarrhea. Antitumor activity was observed in patients with breast cancer who had previous treatment with trastuzumab, anthracyclines, and taxanes, and tumors with a baseline ErbB-2 IHC staining intensity of $2+$ or $3+$.

Neratinib has demonstrated a potent activity against trastuzumabresistant and trastuzumab-naive HER2-positive breast cancer in a phase II study that evaluated the safety and efficacy of a daily $240 \mathrm{mg}$ oral dose on 136 women with locally advanced or metastatic breast cancer (stage IIIB, IIIC or IV). The 16-week progression-free survival (PFS) rate was the primary end point. Secondary end points included safety, objective response rate and clinical benefit rate. Patients were assigned to one of two study arms based on prior treatment with trastuzumab. The efficacy analysis included 127 evaluable patients, 61 in arm $A$ and 66 in arm B. In patients who were previously treated with trastuzumab ( $\operatorname{arm~A}$ ), the 16-week PFS rate was $60 \%$, and the median PFS was 23 weeks. The objective response rate was $26 \%$, and the clinical benefit rate was $36 \%$.

At the present time, several phase II studies of neratinib in combination with chemotherapy (capacitabine, vinorelbine, paclitaxel and doxorubicin) are ongoing, some of them already finished. The tolerance and efficacy will be presented. A large worldwide randomized phase III study of the combination of trastuzumab versus neratinib plus paclitaxel in chemonaïve advanced HER2-positive breast cancer patients will start very soon in order to determine the efficacy of neratinib compared with standard treatment.

References

1. Wong KK, Fracasso PM, Bukowski RM, Lynch TJ, Munster PN, Shapiro GI, Jänne PA, Eder JP, Naughton MJ, Ellis MJ, et al.: A phase I study with neratinib (HKI-272), an irreversible pan ErbB receptor tyrosine kinase inhibitor, in patients with solid tumors. Clin Cancer Res 2009, 15:2552-2558.

2. Burstenin HJ, Sun Y, Tan AR, Dirix L, Vermette JJ, Powell C, Zacharchuk C, Badwe RA: Neratinib (HKI-272), an irreversible pan erbB receptor tyrosine kinase inhibitor: phase 2 results in patients with advanced HER2 ${ }^{+}$breast cancer [abstract 37 ]. SABCS 2008. 


\section{SYMPOSIUM IX}

\section{New targets and new drugs}

\section{S20}

\section{Future role of bevacizumab in breast cancer PF Conte, S Giovannelli}

Department of Oncology and Hematology, University of Modena and Reggio Emilia, Modena, Italy

Breast Cancer Research 2009, 11(Suppl 1):S20 (doi: 10.1186/bcr2281) In the past years, anti-angiogenic therapies have rapidly developed for many solid tumors, including breast cancer.

The first phase III trial of bevacizumab in combination with capecitabine versus capecitabine alone was conducted in 462 patients with MBC previously treated with antracycline or taxane: although the addition of bevacizumab did not improve PFS, there was an absolute increase of approximately $11 \%$ in ORR (20 vs $9 \% ; P=0.001$ ) [1]. In the E2100 trial the addition of bevacizumab to first-line weekly paclitaxel resulted in doubling of both the objective RR $(36.9$ vs $21.2 \% ; P<0.0001)$ and median PFS ( 11.8 vs 5.9 months; $P<0.0001$ ), compared with paclitaxel alone in more than 700 HER2-negative MBC patients, although there was no impact on OS [2]. These findings were corroborated by more complete analyses for regulatory purpose and confirmed by IRF. The AVADO phase III trial compared bevacizumab with placebo in combination with docetaxel as first-line chemotherapy for patients with HER2-negative locally recurrent breast cancer or MBC, showing a significant improvement in PFS (8.8 months for bevacizumab $15 \mathrm{mg} / \mathrm{kg}$ vs 8 months for docetaxel; $P<0.0001$ ) [3]. These results have led to the development of multiple phase III trials of bevacizumab in combination with chemotherapy agents in first-line MBC, along with trials investigating the addition of bevacizumab to other anticancer therapies such as trastuzumab and endocrine agents.

To date no predictive biomarkers for benefit from bevacizumab have been identified; several ongoing trials incorporate molecular studies with the aim of targeting the correct subset of patients (such as triplenegative patients) for bevacizumab therapy.

Clinical trials are underway to evaluate the use of bevacizumab in the adjuvant and neoadjuvant setting. The BEATRICE phase III trial will assess the benefit of adding bevacizumab to standard adjuvant chemotherapy (anthracycline \pm taxane or taxane only) in triple-negative early breast cancer. The addition of bevacizumab to adjuvant chemotherapy (docetaxel/carboplatin or docetaxel-FEC) and trastuzumab will be studied in a further phase III trial (BETH) in patients with HER2positive breast cancer. Other phase III adjuvant trials are evaluating the addition of bevacizumab to standard chemotherapy in HER2-negative and in node-positive or high-risk breast cancer.

The NSABP B-40 ongoing phase III trial will evaluate the addition of bevacizumab to either neoadjuvant docetaxel, docetaxel plus capecitabine or docetaxel plus gemcitabine followed by doxorubicin plus cyclophosphamide in patients with operable breast cancer. The phase III GeparQuinto trial will evaluate the integration of bevacizumab, everolimus and lapatinib into current neoadjuvant regimens [4].

Bevacizumab will probably have an established role in the treatment of some subgroups of MBC and as adjuvant therapy in early breast cancer; we need to identify which subgroups of patients may specifically benefit from bevacizumab therapy.

References

1. Miller KD, Holmes FA, et al:: Randomised phase III trial of capecitabine compared with bevacizumab plus capecitabine in patients with previously treated metastatic breast cancer. $J$ Clin Oncol 2005, 23:792-799.

2. Miller KD, Gralow J, et al:: Paclitaxel plus bevacizumab versus paclitaxel alone for metastatic breast cancer. $N$ Engl J Med 2007, 357:2666-2676.

3. Miles D, Romieu G, et al.: Randomized, double-blind, placebocontrolled, phase III study of bevacizumab with docetaxel or docetaxel with placebo as first-line therapy for patients with locally recurrent or metastatic breast cancer (mBC): AVADO. $J$ Clin Oncol 2008, 26:Abstract 1011.

4. Sirohi B, Smith $\mathrm{K}$, et al:: Bevacizumab in the treatment of breast cancer. Expert Rev Anticancer Ther 2008, 8:1559-1568.

\section{S21}

\section{nab-Paclitaxel (Abraxane ${ }^{\circledR}$ ): an albumin-bound cytotoxic exploiting natural delivery mechanisms into tumors}

J Iglesias

Abraxis Bioscience, Mississauga, ON, Canada

Breast Cancer Research 2009, 11(Suppl 1):S21 (doi: 10.1186/bcr2282)

Taxanes (paclitaxel and docetaxel) are highly active chemotherapeutic agents in the treatment of breast cancer. Being hydrophobic, taxanes require solvents (Cremphor EL or polysorbate) to enable parenteral administration. These solvents contribute to the main toxicities seen with taxanes (hypersensitivity, peripheral neuropathy, and myelosuppression). Cremophor EL can also leach plasticizers from polyvinyl chloride tubing, which can result in severe, sometimes fatal, anaphylactic reactions. To prevent or limit the onset of hypersensitivity reactions, corticosteroids and antihistamines are standard premedication with taxanes. Furthermore, Cremophor EL entraps paclitaxel into circulating micelles, which reduces its availability and delivery into tumors [1]. Micelle formation with solvent-based paclitaxel results in nonlinear kinetics and the absence of a dose-response relationship: increasing the dose increases toxicity without an accompanying enhancement in efficacy.

nab-Paclitaxel is a solvent-free, albumin-bound nanoparticle formulation of paclitaxel that takes advantage of the increased delivery of albumin to tumors through receptor-mediated transport called transcytosis. nab-Paclitaxel binds to gp60, the albumin receptor on endothelial cells, which in turn activates caveolin-1 and the formation of caveolae. Caveolae transport the albumin-paclitaxel conjugate to the extracellular space, including the tumor interstitium. In the tumoral interstitium, SPARC (secreted protein, acidic and rich in cysteine) is selectively secreted by the tumors and binds to albumin-bound paclitaxel with the resultant release of paclitaxel in the vicinity of tumor cells. Together the absence of solvents and the receptor-mediated delivery result in decreased toxicity and increased antitumor activity of nab-paclitaxel compared with solvent-based paclitaxel [2].

nab-Paclitaxel $\left(\right.$ Abraxane ${ }^{\circledR}$ ) has been approved for the treatment of metastatic breast cancer (MBC), based on a phase III trial in 460 patients comparing $260 \mathrm{mg} / \mathrm{m}^{2}$ nab-paclitaxel administered over 30 minutes every 3 weeks (O3W) without premedication with $175 \mathrm{mg} / \mathrm{m}^{2}$ solvent-based paclitaxel given over 3 hours Q3W with premedication. Overall response rates were $33 \%$ and $19 \%$ and the median time to progression was 23.0 weeks and 19.6 weeks, respectively. A significant difference was reported in overall survival in patients receiving nab-paclitaxel versus solvent-based paclitaxel in $\geq 2$ nd lines of treatment (56.4 vs 46.7 weeks, respectively) [3].

Weekly schedules of nab-paclitaxel have proven more effective than Q3W schedules in a randomized phase II study in MBC. Furthermore, outcomes were more favorable for weekly nab-paclitaxel than for 100 $\mathrm{mg} / \mathrm{m}^{2}$ docetaxel administered Q3W, with significantly less toxicity [4]. Development of weekly nab-paclitaxel continues in MBC, NSCLC, melanoma, and pancreatic cancer.

References

1. van Tellingen O, Huizing MT, Panday VR, Schellens JH, Nooijen WJ, Beijnen JH: Cremophor EL causes (pseudo-) non-linear pharmacokinetics of paclitaxel in patients. Br J Cancer 1999, 81:330-335

2. Desai N: Nab technology: a drug delivery platform utilizing endothelial gp60 receptor-based transport and tumourderived SPARC for targeting. Drug Delivery Report. 16th ed. 2007/2008:37-41.

3. Gradishar WJ, Tjulandin S, Davidson N, et al.: Phase III trial of nanoparticle albumin-bound paclitaxel compared with polyethylated castor oil-based paclitaxel in women with breast cancer. J Clin Oncol 2005, 23:7794-7803.

4. Gradishar WJ, Krasnojon D, Cheporov S, et al:: Randomized comparison of nab-paclitaxel weekly or every 3 weeks compared to docetaxel every 3 weeks as first-line therapy in patients with metastatic breast cancer. Presented at the 6th European Breast Cancer Conference (EBCC); April 15-19, 2008, Berlin, Germany [poster 409]. J Clin Oncol 2009, in press. 


\section{CLOSING LECTURE}

\section{$\mathbf{S 2 2}$}

Molecular mechanisms of resistance to HER2-targeted therapy

\section{FJ Esteva}

The University of Texas MD Anderson Cancer Center, Houston, TX, USA Breast Cancer Research 2009, 11(Suppl 1):S22 (doi: 10.1186/bcr2283) The human epidermal growth factor receptor 2 (HER2, also known as ErbB-2) is amplified in 20 to $25 \%$ of invasive breast cancers. HER2 overexpression has been associated with poor survival rates [1]. Trastuzumab (Herceptin) is a monoclonal antibody directed against domain IV of the extracellular domain of HER2. Lapatinib (Tykerb) is a small-molecule tyrosine kinase inhibitor targeted against the intracellular domain of HER2 (ATP binding site). Both agents produce objective response rates in 20 to $30 \%$ of patients with HER2-positive metastatic breast cancer. Efficacy is improved when these targeted therapies are combined with chemotherapy or endocrine therapy. However, the majority of patients that respond to trastuzumab or lapatinib develop progressive disease within 1 year of treatment initiation. Trastuzumab has been shown to improve survival rates in women with early-stage breast cancer. Approximately $15 \%$ of patients develop metastatic breast cancer despite adjuvant trastuzumab, chemotherapy and endocrine therapy [2]. Potential molecular mechanisms of HER2-targeted therapy include increased signaling via the phosphatidylinositol 3-kinase/protein kinase B (PI3K) pathway through overexpression or cross-talk between HER2 and the insulin-like growth factor-I receptor or PTEN loss. Decreased interaction between trastuzumab and HER2 may be caused by steric hindrance of the HER2 receptor by MUC-4, or by the presence of a truncated HER2 protein, which may block inhibitory actions of trastuzumab [3]. One of the mechanisms of action of trastuzumab is the induction of antibodydependent cellular cytotoxicity, which may play an important role in the adjuvant setting. In patients with metastatic breast cancer, continuation of trastuzumab beyond progression resulted in prolongation of the time to progression [4]. The mechanism of action of trastuzumab in this setting is not known. Novel therapies targeted against these aberrant molecular pathways are being studied in laboratory and clinical settings, and offer hope that the efficacy and duration of response to trastuzumab can be greatly improved. These include pertuzumab, a monoclonal antibody that targets domain II of the HER2 extracellular domain and prevents heterodimer formation between HER2 and HER3 or EGFR; trastuzumab-DM1, a potent antibody-drug conjugate that is effective in the setting of resistance to multiple lines of HER2-directed therapy; Hsp90 inhibitors that degrade the HER-2 protein (for example, 17-AAG); irreversible small molecule tyrosine kinase inhibitors (for example, HKI-272); and IGF-IR inhibitors. Indirect approaches include immunotherapy and anti-angiogenic therapy. Understanding mechanisms of resistance in vivo will help us identify what is the optimal treatment for individual patients.

References

1. Slamon DJ, Clark GM, Wong SG, Levin WJ, Ullrich A, McGuire WL: Human breast cancer: correlation of relapse and survival with amplification of the HER-2/neu oncogene. Science 1987, 235:177-182.

2. Romond EH, Perez EA, Bryant J, Suman VJ, Geyer CE, Jr, Davidson NE, Tan-Chiu E, Martino S, Paik S, Kaufman PA, et al:: Trastuzumab plus adjuvant chemotherapy for operable HER2positive breast cancer. N Engl J Med 2005, 353:1673-1684.

3. Nahta R, Yu D, Hung MC, Hortobagyi GN, Esteva FJ: Mechanisms of disease: understanding resistance to HER2-targeted therapy in human breast cancer. Nat Clin Pract Oncol 2006, 3:269-280.

4. von Minckwitz G, du Bois A, Schmidt M, Maass N, Cufer T, de Jongh FE, Maartense E, Zielinski C, Kaufmann M, Bauer W, et al:: Trastuzumab beyond progression in human epidermal growth factor receptor 2-positive advanced breast cancer: a German Breast Group 26/Breast International Group 03-05 study. J Clin Oncol 2009, 27:1999-2006.

\section{POSTERS}

P1

\section{Gail's model as first step for early diagnosis: National Cancer Institute of Naples experience}

I Capasso', E Esposito', M Montella², A Crispo², M Grimaldi2, M D'Aiuto', G Beneduce ${ }^{3}$, G Esposito ${ }^{3}$, G D'Aiuto' $^{1}$

${ }^{1}$ National Cancer Institute Dip Senologia, Naples, Italy; ${ }^{2}$ National Cancer Institute Div Epidemiologia, Naples, Italy; ${ }^{3}$ National Cancer Institute Medicina di Laboratorio, Naples, Italy

Breast Cancer Research 2009, 11(Suppl 1):P1 (doi: 10.1186/bcr2284)

Objective We started up an intensive program of clinical-instrumental surveillance according to Gail's model. The aim was to increase cases of early diagnosis in women at higher risk.

Methods For 568 patients, Gail's model risk was evaluated and clinical examination, mammography, and ultrasonography were performed.

Results According to Gail's model parameters among 568 patients: the mean age of menarche was 12.5 years; according to family history for breast cancer, 43\% (244 patients) had a first-degree relative, $24 \%$ (136 patients) had a second-degree relative, and 33\% (188 patients) had no family history; classes of age most represented were 41 to 50 years (30\%) and 51 to 60 years $(27 \%)$; the mean age of first live birth was 25 years; and according to number of biopsies, 326 patients had previous breast biopsies - 29 patients (9\%) positive for atypical hyperplasia and 297 patients (91\%) positive for nonatypical hyperplasia. Combining family history with previous breast biopsies showed that, of 380 patients with family history of breast cancer, 154 patients $(41 \%)$ underwent biopsy and the highest risks were noted in the age class of 51 to 60 years (107 patients).

Conclusions Thanks to an intensive clinical-instrumental management considering Gail's model, in our breast cancer risk ambulatory program we reached early diagnosis in 13 cases $(8.4 \%)$, and obtained a reduction of anxiety in women that felt more protected. Gail's model represents the first step toward achieving breast cancer control, particularly paying attention to the relationship between family history and previous biopsies.

\section{P2}

Metabolic syndrome, hyperinsulinaemia and body mass index as risk factors in breast cancer: National Cancer Institute of Naples experience I Capasso', E Esposito', M Montella², A Crispo², M Grimaldi², M D'Aiuto', G Beneduces ${ }^{3}$, G Esposito ${ }^{3}$, M De Marco4, G D'Aiuto'

${ }^{1}$ National Cancer Institute Dip Senologia, Naples, Italy; ${ }^{2}$ National Cancer Institute Div Epidemiologia, Naples, Italy; ${ }^{3}$ National Cancer Institute Medicina di Laboratorio, Naples, Italy; ${ }^{4}$ National Cancer Institute, Naples, Italy

Breast Cancer Research 2009, 11(Suppl 1):P2 (doi: 10.1186/bcr2285)

Objective Metabolic syndrome appears to be connected to the onset of breast cancer through two pathways: obesity determines a high concentration of aromatase; and also insulin resistance, the related hyperinsulinaemia and high levels of IGF-1 (which rules as a growth factor like gonadotropic factor creating a hyperoestrogenic state). The goal we aim to reach is to identify a group at higher risk of developing breast cancer and to provide them with lifestyle models in order to support primary prevention and to assist the lead time in breast cancer detection.

Methods We set up a project stratifying women $\geq 35$ years old into three groups: women with borderline lesions and/or with familiarity; women with breast cancer; and healthy women without any breast pathology. Each woman, after consent, completed a questionnaire about personal and familial anamnesis and physical activity. For each woman, blood pressure, body mass index and waist-hip ratio were measured. Blood samples were taken in order to determine glycaemia, cholesterolaemia, triglyceride, and insulinaemia. Clinical-instrumental management was performed. 
Results Two hundred and fifty women have been enrolled, stratified and studied, as described previously, and we are evaluating whether they are affected by metabolic syndrome and how it impacts on the onset of breast cancer.

Conclusions Metabolic syndrome can be considered an important risk factor in developing breast cancer. Weight control, reduction of insulin seric levels and a correct lifestyle should be praised as efficient instruments to prevent breast carcinoma.

\section{P3}

\section{HER2 assessment using fluorescence in situ hybridization compared with Oncotype DX and association with risk of breast cancer death} FL Baehner ${ }^{1}$, NS Achacoso'2, T Maddala ${ }^{3}$, C Alexander ${ }^{3}$, S Shak ${ }^{3}$, CP Quesenberry², LC Goldstein ${ }^{4}$, AM Gown ${ }^{4}$, LA Habel ${ }^{2}$

1 University of California, San Francisco, CA, USA; ${ }^{2}$ Kaiser Permanente, Oakland, CA, USA; ${ }^{3}$ Genomic Health Inc., Redwood City, CA, USA; ${ }^{4}$ PhenoPath, Seattle, WA, USA

Breast Cancer Research 2009, 11(Suppl 1):P3 (doi: 10.1186/bcr2286)

Objective Guidelines mandate demonstration of $95 \%$ concordance to another laboratory or method before reporting patient HER2 results. In this analysis, HER2 results obtained with Oncotype DX, a validated 21gene recurrence score assay utilizing RT-PCR, were compared with standard fluorescence in situ hybridization (FISH) results. The Oncotype DX HER2 results were then associated with relative risk of breast cancer death.

Methods Breast cancer specimens from the Kaiser Oncotype DX study were evaluated for HER2 by FISH with positive $>2.2$, equivocal 1.8 to 2.2 , and negative $<1.8$. HER2 was also assessed with Oncotype DX (RT-PCR) with positive $\geq 11.5$ units, equivocal $>10.7$ to $<11.5$ units, and negative $\leq 10.7$ units (each unit $=$ twofold change in expression). Concordance analyses were conducted following ASCO/CAP guidelines that mandate 95\% concordance. Logistic regression was used to estimate association between Oncotype DX HER2 results and risk of breast cancer death.

Results Of 568 patients, $12 \%$ (67 patients) were HER2+ by Oncotype DX and $11 \%(60)$ by FISH. Fifty-five patients were HER2 ${ }^{+}$by both methods. Of the 12 patients Oncotype DX HER2 ${ }^{+}$but FISH negative, using FISH 11 patients were HER2 ${ }^{-}$and one patient was HER2 equivocal. The positive and negative HER2 concordance by FISH and Oncotype DX was $97 \%(95 \% \mathrm{Cl}=96 \%$ to $99 \%)$. Risk of breast cancer death was significantly greater in patients with HER2 $\geq 11.5$ (OR $=1.84,95 \% \mathrm{Cl}=1.13$ to 2.99$)$ compared with patients with HER2 $\leq 10.7$. However, in patients with HER2 $>10.7$ to $<11.5$ compared with HER2 $\leq 10.7$, there was no greater risk (OR $=0.75$, $95 \% \mathrm{Cl}=0.46$ to 1.20$)$. There were $71(12.5 \%)$ polysomy cases. Conclusions There is a high degree of concordance between RT-PCR using Oncotype DX and central laboratory FISH assessment of HER2 status.

Acknowledgement Supported by funding from Genomic Health Inc. Disclosure FLB is an employee and shareholder of Genomic Health, Inc.

\section{P4}

\section{Population-based study of hormone receptor status comparing Oncotype DX with standard immunohistochemistry}

FL Baehner', T Maddala'2, C Alexander², AM Gown ${ }^{3}$, LC Goldstein 3 , NS Achacoso ${ }^{4}$, S Shak ${ }^{2}$, CP Quesenberry 4 , LA Habel ${ }^{4}$

${ }^{1}$ University of California, San Francisco, CA, USA; ${ }^{2}$ Genomic Health Inc., Redwood City, CA, USA; ${ }^{3}$ PhenoPath, Seattle, WA, USA; ${ }^{4}$ Kaiser Permanente, Oakland, CA, USA

Breast Cancer Research 2009, 11(Suppl 1):P4 (doi: 10.1186/bcr2287)

Objective Accurate measurement of hormone receptor (HR) status is important as hormonal therapy reduces risk of recurrence by $>50 \%$ in breast cancer patients with hormone-sensitive tumors. We compared estrogen receptor (ER) and progesterone receptor (PR) results obtained with Oncotype DX, a validated 21-gene recurrence score assay that utilizes quantitative RT-PCR, with results from established immunohistochemistry (IHC).

Methods Breast cancer specimens from the Kaiser Oncotype DX study were evaluated by IHC for ER (SP1) and PR (636) using $\geq 1 \%$ staining for positivity. ER and PR status was also determined using Oncotype DX (RT-PCR) with pre-defined positivity cutoff values of 6.5 units and 5.5 units, respectively (each unit $=$ twofold change in expression). HR positivity was defined as $\mathrm{ER}^{+}$and/or $\mathrm{PR}^{+}$.

Results The overall concordance in 607 evaluable patients $(95 \% \mathrm{Cl})$ between IHC and Oncotype DX was $96 \%$ for ER, $90 \%$ for PR, and $95 \%$ for HR (all $P<0.0001)$. The kappa $(95 \% \mathrm{Cl})$ between IHC and Oncotype DX was $83 \%$ for ER, $76 \%$ for PR, and $81 \%$ for HR (all $P<0.0001)$. For discordant pairs that were within cutoff limits, there were 20 that were IHC ER- but ER+ by Oncotype DX, 17 (85\%) of which were within cutoff limits; another 38 were IHC PR- but $\mathrm{PR}^{+}$by Oncotype DX, $23(61 \%)$ of which were within cutoff limits; seven pairs were $\mathrm{IHC} \mathrm{ER}^{+}$but $\mathrm{ER}^{-}$by Oncotype $\mathrm{DX}$, six (86\%) of which were within cutoff limits; and 22 pairs were IHC PR+ but Oncotype DX PR-, $19(86 \%)$ of which were within cutoff limits.

Conclusions There was a high degree of concordance between Oncotype DX and standard IHC methods for ER, PR and HR status, indicating Oncotype DX may be a valid alternative to IHC. The notable incidence of IHC HR-negative patients who were Oncotype DX HRpositive, which could affect treatment decisions, deserves further study. Acknowledgement Supported by funding from Genomic Health Inc. Disclosure FLB is an employee and shareholder of Genomic Health, Inc.

\section{P5}

\section{Breast cancer presenting as axillary abscess and unilateral lymphedema \\ R Lakshmanan, A Cheng}

Department of General Surgery, Alexandra Hospital, Singapore

Breast Cancer Research 2009, 11(Suppl 1):P5 (doi: 10.1186/bcr2288)

Introduction This is a case report illustrating the initial presentation of breast cancer as an axillary abscess and ipsilateral arm lymphedema.

Case report A 62-year-old Indian lady presented with the main complaints of left arm swelling and pain of 6 months duration. Clinical examination revealed gross right upper limb lymphedema; associated with tender axillary abscess with possibility of infected lymphadenopathy. Right breast examination revealed multifocal hard breast lumps with a retracted nipple. Ultrasound and mammography of the breasts showed an edematous right breast with several suspicious nodules. As the patient was septic and symptomatic from the axillary abscess, we performed an axillary drainage and axillary lymph node biopsy, which proved positive for adenocarcinoma. Ultrasound-guided core biopsy of the breast lesion was invasive ductal carcinoma. The estrogen and progesterone receptors were negative, c-erb2 was positive. Metastatic work-up showed that the patient had bilateral pulmonary metastases with bony metastases as well.

Conclusions The present case highlights the fact that, despite a breast screen programme established in Singapore, there are patients presenting with locally advanced cancers. This case is particularly interesting as she presented as a surgical emergency for an axillary abscess.

\section{P6}

Epidemiological changes in premenopausal patients with breast cancer: a two-decade retrospective study A Garcia-Faura1,2, C Santiago $0^{1,2}$, L Marquès ${ }^{1,2}$

${ }^{1}$ Department of Obstetrics and Gynecology, Institut Marquès, Barcelona, Spain; ${ }^{2}$ Fundació Leonardo Marquès, Barcelona, Spain Breast Cancer Research 2009, 11(Suppl 1):P6 (doi: 10.1186/bcr2289)

Introduction In the past decades there appears to have been a decrease in the mean age of presentation of breast cancer in premenopausal patients from developed countries, coinciding with an increase in maternal age at first delivery. The main objectives of this 
study were to determine the epidemiological features of premenopausal patients with primary breast carcinoma diagnosed in our unit for the past 20 years, and to evaluate any significant changes observed during this period.

Methods Between January 1988 and December 2008, 162 premenopausal patients with primary breast carcinoma were evaluated at our center. A total of 85 patients came prior to January 2000 , and 77 patients between January 2000 and December 2008. We used statistical parametrical tests (Student $t$ test and chi-square test) to compare mean age at the time of diagnosis, maternal age at first delivery, parity, lapse time between last delivery and diagnosis of cancer, staging, histological tumor grade, and whether the diagnosis was made based on on-demand screening mammography or based on clinical findings.

Results No significant differences were found in female age at the time of diagnosis $(43.1 \pm 5.2$ years vs $43.6 \pm 4.6$ years) There were significant differences in maternal age at first pregnancy $(27.2 \pm 4.6$ years vs $31.5 \pm 4.4$ years), lapse time between last delivery and cancer (11.9 \pm 6.3 years vs $9.3 \pm 6.1$ years), parity $(54 \%$ vs $45 \%$ in multiparous patients), staging ( $47 \%$ vs $56 \%$ stage 0 or I), histological grade ( $26 \%$ vs $42 \%$ grade III tumors) and diagnosis ( $43 \%$ vs $57 \%$ by screening).

Conclusions The results of this study indicate that female age at the time of diagnosis has remained constant for the past two decades while maternal age for first delivery has increased. The lapse time until cancer diagnosis decreased from the last delivery. In the past decade, on-demand screening programs have helped us diagnose more cases that were at lower stages even if they were of higher histological grade.

\section{P7}

\section{Breast carcinoma: molecular markers and subtypes to predict patients at risk of developing metastatic disease}

\section{CR Inderhaug, LS Murillo, MJ Webber, GM Callagy}

Department of Pathology, National University of Ireland, Galway, Ireland

Breast Cancer Research 2009, 11(Suppl 1):P7 (doi: 10.1186/bcr2290)

Objective To identify molecular markers and molecular subtypes in breast carcinoma that predict patients at higher risk of developing bone, brain, and visceral metastasis.

Methods Immunohistochemical analysis using a panel of antibodies against ER, PR, Her2, EGFR, CK5/6, CK14, Ki67, E-cadherin, Bcl2 and p53 was performed on tissue microarray sections of breast carcinoma with bone, brain and visceral metastasis $(33,13$ and 30 cases) and 483 cases of breast carcinoma without metastasis. Using standard methods of scoring, the expression levels of receptors and subtype distribution (luminal A, luminal B, Her2, basal, triple negative) were compared within these groups.

Results When compared with breast tumours without metastases, bone metastases were significantly associated with ER $(P=0.01)$ and E-cadherin $(P=0.014)$ positive breast tumours, brain metastases were significantly associated with ER $(P=0.01)$ and $\operatorname{PR}(P=0.001)$ positive breast tumours, and visceral metastases were significantly associated with $\mathrm{ER}(P<0.0001), \mathrm{PR}(P=0.013)$, Ki67 $(P=0.009)$, EGFR $(P=0.01)$ and p53 $(P=0.002)$ positive breast tumours. The triple-negative subtype was significantly associated with breast tumours with bone $(P=0.001)$ and brain $(P<0.001)$ metastases, while the basal subtype was significantly associated with breast tumours with visceral metastases $(P=0.005)$, when compared with breast tumours without metastatic disease. There was a significant association with tumour size $>2 \mathrm{~cm}$ in breast tumours with bone $(P=0.021)$ and visceral metastases $(P<0.001)$, compared with breast tumours without metastatic disease.

Conclusions We have shown that, using immunohistochemistry, a standard panel of molecular markers of breast carcinoma can be of significant value in predicting sites of metastases.
P8

How many sentinel lymph nodes should we excise? J Piechocki ${ }^{1}$, S Mazur', WP Olszewski², I Kozłowicz-Gudziñska ${ }^{3}$, E Topwik'

${ }^{1}$ Breast Cancer \& Reconstructive Surgery Clinic, ${ }^{2}$ Department of Pathology, and ${ }^{3}$ Department of Nuclear Medicine, Maria Skłodowska Curie Memorial Cancer Center, Warsaw, Poland

Breast Cancer Research 2009, 11(Suppl 1):P8 (doi: 10.1186/bcr2291)

Objective Radioactivity may be detected over several nodes during sentinel node biopsy (SNB), thus indicating that they all should be excised for examination. Biopsy of an insufficient number of lymph nodes (LNs) may produce a false negative result, while excision of too many contradicts the SNB idea itself, turning it into an incomplete lymphadenectomy. We aim at establishing the minimal number of LNs that must be removed without compromising the reliability.

Materials and methods During years 2004 to 2008, 1,478 SNBs were performed in our department. Invasive cancer was diagnosed in 1,202 cases and these were included in our present study. LNs were removed one by one, according to the expressed radioactivity, until the last node showed radioactivity below $10 \%$ of the previous one. All LNs were marked in order of their expressed radioactivity and subsequent removal.

Results Metastases were found in 228/1,202 (19\%) patients with invasive breast cancer. The postoperative full pathologic report showed that $143 / 228$ patients $(62.7 \%)$ had no $L N$ metastases other than to sentinel LNs; they were, however, found in the remaining $37.3 \%$. The number of excised sentinel nodes varied from one to nine, the average was 2.04. Table 1 presents groups of patients with particular numbers of sentinel LNs excised and percentages of metastases found. In our experience, almost full (97\%) information about the presence of metastases could be based on the biopsy of three first sentinel nodes, and in full (100\%) of five first sentinel nodes.

\section{Table 1 (abstract P8)}

\begin{tabular}{lccc}
\hline $\begin{array}{l}\text { Number of } \\
\text { sentinel lymph } \\
\text { nodes }\end{array}$ & $\begin{array}{c}\text { Number of } \\
\text { patients }\end{array}$ & $\begin{array}{c}\text { Total number } \\
\text { of sentinel } \\
\text { lymph nodes }\end{array}$ & $\begin{array}{c}\text { Number of } \\
\text { sentinel lymph } \\
\text { nodes with } \\
\text { metastases }\end{array}$ \\
\hline 1 & 105 & 105 & 105 \\
2 & 78 & 156 & 81 \\
3 & 35 & 105 & 36 \\
4 & 2 & 8 & 4 \\
5 & 2 & 10 & 2 \\
6 & 3 & 18 & 0 \\
7 & 1 & 7 & 0 \\
8 & 1 & 8 & 0 \\
9 & 1 & 9 & 0 \\
Summary & 228 & & \\
\hline
\end{tabular}

Conclusions In most cases, full information about the presence of metastases to sentinel nodes could be obtained from the biopsy of the first three LNs, and $100 \%$ information of the first five. Biopsy of more than five LNs proved to be of no additional value.

P9

Prognostic value HER-2/neu expression in T1 to T3 breast cancer with axillary lymph node metastasis s Maksimovic

General Hospital 'Sveti Vracevi', Bijeljina, Bosnia and Herzegovina Breast Cancer Research 2009, 11(Suppl 1):P9 (doi: 10.1186/bcr2292)

Objective Studies on the association of HER-2/neu with the axillary lymph node metastasis are controversial. Amplification of the protein 
product of the HER-2/neu oncogene in primary breast cancer specimens is associated with an adverse prognosis.

Methods From January 2000 to December 2008, 504 breast cancer patients were operated on at General Hospital 'Sveti Vracevi' in Bijeljina. We selected $253(50.2 \%)$ patients with breast cancer who had metastases to axillary lymph nodes.

Results Extracapsular extension (ECM) was found in 103 (40.7\%) patients. The patients were identified and divided into two groups: the HER-2-positive group (38 patients) and the HER-2-negative group (65 patients). In the HER-2-positive group ECM was seen in $62.5 \%$ of patients, compared with $37.4 \%$ in the HER-2-negative group $(P=0.059)$. The total number of lymph nodes showing ECM were also significantly more in the HER-2-positive group (48 out of $81,59.25 \%$ ), versus 13 out of $60(21.66 \%)$ in the HER-2-negative group $(P<0.001)$. With a median follow-up of 96 months, factors with independent prognostic value for disease-free survival by multivariate analysis included HER-2/neu overexpression with extracapsular extension $(P<0.005)$, pN category $(P<0.01)$, presence of lymphovascular invasion $(P<0.005)$, and ECM $(P<0.001)$. An independent negative prognostic effect on overall survival was observed for HER-2/ neu overexpression with extracapsular extension $(P<0.05), \mathrm{pN}$ category $(P<0.05)$, and presence of lymphovascular invasion $(P<0.005)$ and ECM $(P<0.001)$.

Conclusions In patients whose tumors expressed HER-2/neu who had positive lymph nodes and extracapsular extension, prognosis was significantly worse compared with those who were HER-2/neunegative and lymph node-positive with extracapsular extension. These findings have led to the conclusion that HER-2/neu overexpression is associated with a more aggressive subtype of cancer.

\section{P10}

\section{Role of p27 in tamoxifen response in breast cancer cell lines}

\section{S Nilsson ${ }^{1}$, C Holm 1 , G Landberg 1 ,2}

${ }^{1}$ Center for Molecular Pathology, Department of Laboratory Medicine, Malmö University Hospital, Lund University, Malmö, Sweden; ${ }^{2}$ Breakthrough Breast Cancer Research Unit, Paterson Institute for Cancer Research, Manchester, UK

Breast Cancer Research 2009, 11(Suppl 1):P10 (doi: 10.1186/bcr2293)

Objective The aim of the study was to experimentally point out the clinical results of p27 as a predictive factor for tamoxifen response in estrogen receptor-positive breast cancer cell lines. Normal human mammary epithelium expresses the CDK inhibitor p27, whereas p27 is downregulated in a fraction of breast cancer, associated with poor prognosis and aggressive features. Besides prognostic information, p27 has also been linked to prediction of treatment effects. In a randomized study, where patients were either treated with adjuvant tamoxifen or no treatment, we observed that low p27 was not associated with prognostic information but instead linked to poor treatment effect of the selective estrogen receptor modulator tamoxifen. In this study we evaluated the clinical data with an experimental approach using breast cancer cell lines.

Methods Proliferation and cyclin $D_{1}$ protein levels were monitored after treatment with estrogen alone or together with tamoxifen, and p27 levels were modulated with siRNA transfection.

Results Surprisingly, p27-downregulated MCF-7 cells responded to tamoxifen treatment but showed a decreased sensitivity to estrogen stimulation. For Cama-1 and T-47D cells there was no difference in estrogen or tamoxifen response in relation to $p 27$. Cyclin $D_{1}$ protein levels corresponded to p27 in the siRNA experiments, validating the function of p27 in the model system.

Conclusions Our results indicate that p27 was not required for mediating a tamoxifen effect in the tested cell lines but could function as an assembly factor essential for estrogen-induced proliferation. This potential assembly factor function for p27 might explain the observation in the primary breast cancer of a tamoxifen treatment predictive function for $\mathrm{p} 27$ in breast cancer.

\section{P11}

Inactivation of the retinoblastoma tumour suppressor pathway in premenopausal breast cancer is associated with resistance to tamoxifen

\section{S Lehn 1,2, G Landberg 1,2}

${ }^{1}$ Center for Molecular Pathology, Department of Laboratory Medicine, Lund University, UMAS, Malmö, Sweden; ${ }^{2}$ Breakthrough Breast

Cancer Research Unit, Paterson Institute for Cancer Research, Manchester, UK

Breast Cancer Research 2009, 11(Suppl 1):P11 (doi: 10.1186/bcr2294)

Objective shRNA mediated knockdown of the retinoblastoma tumour suppressor $(p R b)$ in oestrogen receptor $\left(E R^{+}\right)$cell lines leads to resistance to tamoxifen, and $\mathrm{pRb}$ inactivation has further been associated with more aggressive disease. By studying the tamoxifen response in premenopausal patients randomised to either control or tamoxifen treatment, we aim to determine the importance of $\mathrm{pRb}$ inactivation in relation to tamoxifen response.

Methods Breast cancer samples were assembled in tissue microarrays, immunohistochemically stained for phos-pRb and evaluated as the fraction of positive nuclei divided into four groups: $0 \% ; 1$ to $10 \%$; 11 to $25 \%$; and 26 to $100 \%$. pRb-inactivated tumours were defined using the phos- $\mathrm{Rb}$ parameter in combination with the proliferation marker Ki67. Tumours with none or low expression of phos-pRb displaying a high proliferation rate were defined as $\mathrm{pRb}$ inactivated $(n=57)$, whereas the remaining tumours were considered to have a functional pRb pathway $(n=273)$.

Results Inactivation of $\mathrm{pRb}$ was significantly correlated to larger tumours $(P=0.001)$, lymph node-negative disease $(P=0.001)$ and a higher histological grade $(P<0.001)$. There was a positive correlation to cyclin $\mathrm{E}$ levels $(P<0.001)$ but a negative correlation to cyclin $\mathrm{D}_{1}$ $(P<0.001)$ and ER as well as progesterone receptor levels (both $P<0.001)$. A significant difference was noted in recurrence-free survival when comparing no treatment with tamoxifen treatment in the patient group with functional $\mathrm{pRb}(P=0.003)$; however, the beneficial effect of tamoxifen was lost in the pRb-inactivated group $(P=0.619)$. A multivariate analysis confirmed that inactivation of $\mathrm{pRb}$ was significantly associated with impaired tamoxifen response.

Conclusions The functional inactivation of $\mathrm{pRb}$ seems to be part of the explanation to why a subgroup of $\mathrm{ER}^{+}$tumours does not respond to tamoxifen. An evaluation of $\mathrm{pRb}$ status in $\mathrm{ER}^{+}$tumours could therefore possibly contribute to a more effective treatment.

\section{P12}

\section{Risk of breast cancer amongst women who start smoking as teenagers \\ EJ Odiase}

Women Action Initiative against Tobacco, SmokeFree Foundation, Abuja, Nigeria

Breast Cancer Research 2009, 11(Suppl 1):P12 (doi: 10.1186/bcr2295)

Introduction We examined the effect of smoking on breast cancer risk in women who started smoking as teenagers. The women, 30 to 50 years of age who had smoked for at least 20 years, were surveyed through a mailed questionnaire at recruitment. Altogether, $1.34 \%$ of the women were diagnosed with incident, invasive breast cancer. In contrast, women who had smoked for at least 20 years, but started after their first child's birth, did not experience an increased breast cancer risk. Our results support the notion that women who start smoking as teenagers and continue to smoke for at least 20 years may increase their breast cancer risk. Recent studies have shown breast cancer risk amongst women who start to smoke as teenagers, especially those who began before their first child's birth. Ninety percent of all smokers began to smoke before the age of 19 - being the target of the cigarette manufacturers at this tender and immature age. Because of this early start and the addiction from nicotine, it is almost impossible to quit, thereby leaving enough time for the carcinogens present in tobacco smoke to damage the body. 
Figure 1 (abstract P12)

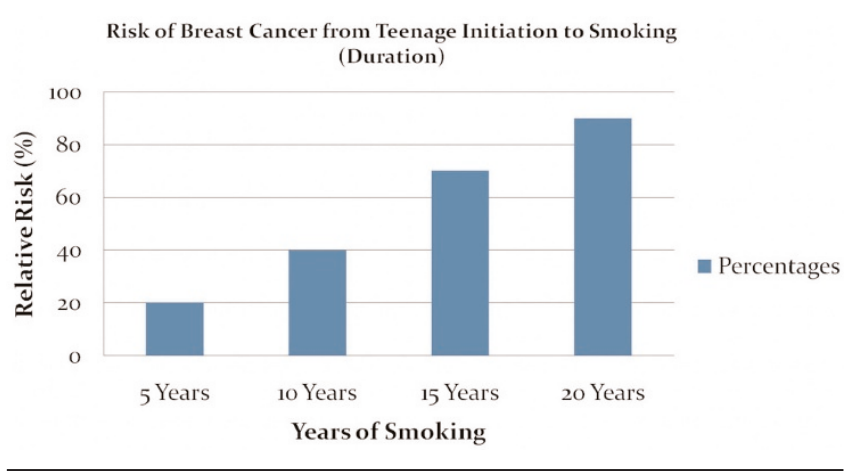

Figure 2 (abstract P12)

Risk of Breast Cancer from Teenage Initiation to Smoking (Intensity)

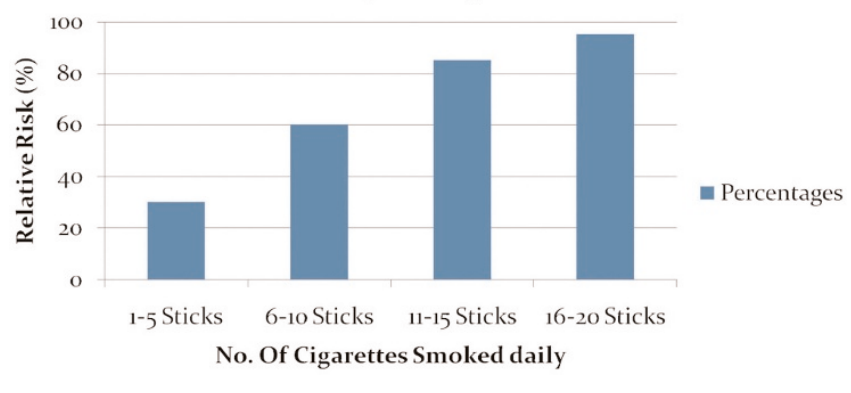

Subjects The number of women used for this study was a stunning 102,098 who completed a mailed questionnaire at recruitment through a period of 9 years (1996 to 2004). All Kenyan-Nigerian women were aged from 30 to 50 .

Methods We estimated the relative risk (RR) of breast cancer associated with different measures of smoking initiation: duration - the period in which the smoker had smoked, which was a strong determinant of the risk; and intensity - the number of cigarettes that were smoked during this period. Cox proportional hazard regression models were used to estimate these risks so as to adjust for confounding variables. We conducted analyses on the entire study population, among women who had smoked for at least 20 years, among nondrinkers, and separately for each country.

Results Altogether, 1,240 women were diagnosed with incident, invasive breast cancer. Compared with women who had never smoked, women who smoked for at least 20 years and who smoked 10 cigarettes or more daily had a RR of $90 \%$. In contrast, women who had smoked for at least 20 years, but started after their first birth, did not experience an increased breast cancer risk (Figure 1).

Discussion The risk of breast cancer is almost double if young women start smoking within 5 years of their first menstrual cycle. Cigarette smoke contains over 40 potent cancer-causing chemicals. Scientists have been able to show that these chemicals can cause breast cancer cells in laboratory cultures to become cancerous. Women who started smoking in their teens developed breast cancer before menopause. One reason for this is that teenage breast tissue is still developing, which makes it more susceptible to the cancer-causing effects of the chemicals in smoke. Another factor that turned out to be important from this study was the amount and duration of smoking. More cigarettes per day and more years of smoking led to a higher chance of breast cancer (Figure 2).

Recommendations From this study we recommend that smoking prevention should be reinforced among adolescents, especially in high in Philadelphia points out that tobacco already has a devastating effect on women by causing lung cancer. Lung cancer and breast cancer are the major causes of cancer deaths in women. The results of this study mean women have even more reasons to avoid tobacco.

Conclusions Our results support the notion that women who start smoking as teenagers and continue to smoke for at least 20 years may increase their breast cancer risk.

\section{P13}

VEGF-A, VEGF-C, VEGF-R2, EGFR and HER2 in serum plus EGFR in tissue of patients with triple-negative breast cancer

R losifidou1, G Galaktidou², A Ananiadis', N Bladika³, F Patakiouta ${ }^{3}$, A Bousoulegas ${ }^{1}$

${ }^{1} 3$ rd Surgical-Breast Clinic, ${ }^{2}$ Department of Clinical Research, and ${ }^{3}$ Pathology Department, Anticancer Hospital Theageneio, Thessaloniki, Greece

Breast Cancer Research 2009, 11(Suppl 1):P13 (doi: 10.1186/bcr2296) Objective Triple-negative breast cancer (ER-PR-HER2-negative) is not very common, has very poor prognosis and its therapeutic approach is a problem. This cancer type overexpresses EGFR in tissue. Elevated VEGF-C levels may be a predictor of lymph node metastases. VEGF-R2 plays an important role in tumor angiogenesis. We measured VEGF-C, VEGF-R2, EGFR, VEGF-A and HER2 in serum and EGFR in tissue. We compared all these parameters to find correlations between them.

Patients and methods Seventy-three patients with triple-negative breast cancer were enrolled in this study. All patients had chemotherapy and radiotherapy after the surgical treatment. All of the parameters were measured in serum by ELISA.

Results VEGF-C, VEGF-A, VEGF-R2 and HER2 in serum were measured in 73 patients. From our results, serum VEGF-C was overexpressed in $77 \%(11,393 \pm 2,160 \mathrm{pg} / \mathrm{ml}$, normal values: 2,459 to $6,651)$. Serum VEGF-R2 was overexpressed in the same patients $(8,948 \pm 1,234 \mathrm{pg} / \mathrm{ml}$, normal values: 2,000 to 6,000$)$. Serum VEGF-A was overexpressed in four patients but we found that they had extremely elevated levels of all of the VEGF agents. EGFR was measured in 54 patients who do not have any recurrence of the disease. It was overexpressed in $21 / 54(>0.13 \mathrm{fmol} / \mathrm{ml})$. In those patients, EGFR was also overexpressed in tissue (57\%). We found also that in patients with overexpression of both serum and tissue EGFR, VEGF-C was not overexpressed but VEGF-R2 was overexpressed. Serum HER2 was overexpressed in eight patients. In those patients, serum and tissue EGFR (HER1) was also overexpressed.

Conclusions We found interesting correlations between these factors. VEGF-C and VEGF-R2 have significant correlation. On the contrary, patients with overexpression of serum and tissue EGFR and VEGF-R2 do not overexpress VEGF-C. We need more patients to evaluate these results as they can help in the search for anti-angiogenic therapies.

\section{P14}

\section{A supernumerary muscle complicated axillary lymphadenectomy: case report \\ T Totlis',2, R losifidou', F Pavlidou', Ath Papanikolaou', K Natsis ${ }^{2}$, A Bousoulegas}

13rd Surgical Department - Breast Unit, 'Theagenio' Cancer Hospital, Thessaloniki, Greece; '2Department of Anatomy, Medical School, Aristotle University, Thessaloniki, Greece

Breast Cancer Research 2009, 11(Suppl 1):P14 (doi: 10.1186/bcr2297) During lymphadenectomy in the left axilla of a 38-year-old woman with a $1.4 \mathrm{~cm}$ invasive ductal breast carcinoma, when the surgeon attempted to prepare the lateral margin of the pectoralis major muscle, an aberrant muscular slip was observed slightly lateral and deep to the pectoralis major muscle. The two muscles were separated by a narrow band of connective tissue that was completely dissected. Following this, the supernumerary muscle located in the center of the surgical field was pulled towards the latissimus dorsi muscle. As a result, the 
muscle formed an arcuate course and the lymphadenectomy was carried out troublesomely through a limited field. Nevertheless, the amount of the lymph node dissected was satisfactory (N: 0/25). Based on the anatomical characteristics of the muscle, it was recognized as a pectoralis quartus muscle. To our knowledge this is the first report of a pectoralis quartus muscle as a surgical finding. The surgeon should be aware of the possible presence of this supernumerary muscle as well as its anatomical characteristics in order to avoid any complications.

\section{P15}

\section{Differential expression of cytoplasmic and stromal $\beta$-arrestin-1 is associated with separate aspects of tumor behavior in breast cancer \\ K Lundgren, K Jirström, G Landberg}

Center for Molecular Pathology, Department of Laboratory Medicine, Malmö University Hospital, Lund University, Malmö, Sweden

Breast Cancer Research 2009, 11(Suppl 1):P15 (doi: 10.1186/bcr2298)

Objective The main focus of our study was to investigate the importance of $\beta$-arrestin- 1 in breast cancer and to elucidate a possible link between $\beta$-arrestin-1 protein overexpression and CCND1 amplification, since both genes map to chromosome locus 11q13, a region often amplified in breast cancer.

Methods The $\beta$-arrestin- 1 protein expression was assessed in two different patient cohorts. The first included 179 premenopausal and postmenopausal breast cancer patients and the second included 500 breast cancer cases from premenopausal patients randomized to either 2 years of tamoxifen or no adjuvant treatment.

Results High cytoplasmic expression of $\beta$-arrestin-1 turned out to be associated with estrogen receptor negativity and HER2 amplification, whereas high $\beta$-arrestin-1 expression in stromal cells was associated with a more aggressive tumor phenotype. In the randomized cohort, cytoplasmic $\beta$-arrestin-1 expression was inversely correlated to amplification of CCND1 and positively correlated to expression of Chk1, previously described as a marker for distal $11 \mathrm{q}$ deletion, implying that the ARRB1 gene, instead of being co-amplified, might be deleted in the 11 q13 amplification event.

Conclusions Interestingly, $\beta$-arrestin-1 may play different roles for tumor cells, depending on whether it is expressed by the tumor cell itself or by the surrounding stroma. In addition, amplification of the CCND1 gene might be associated with a loss of the ARRB1 gene.

\section{P16}

\section{Value of diffusion-weighted imaging in} differentiating benign from malignant breast lesions S Stijven, E Gielen, M Horvath, L Meylaerts

Department of Medical Imaging, Ziekenhuis Oost Limburg, Genk, Belgium

Breast Cancer Research 2009, 11(Suppl 1):P16 (doi: 10.1186/bcr2299) Introduction MRI has a proven high sensitivity for the detection of breast cancer. Besides contrast enhancement which is related to the vascularity of breast tumours, tumour high cellularity can be characterized or better measured via diffusion-weighted imaging (DWI), which is an important characteristic of tumours. DWI, combined with morphology and kinetics of contrast enhancement, may increase the specificity of MRI and may help in differentiating between normal and malignant breast lesions.

Materials and methods So far, 11 patients with biopsy-proven breast cancer and eight patients with benign breast lesions underwent DWI of the breast with an echo-planar imaging (EPI) sequence at $1.5 \mathrm{~T}$. The apparent diffusion coefficient (ADC) was measured using four different b-values: $0,300,500$, and 800 seconds $/ \mathrm{mm}^{2}$.

Results The $A D C$ values of the malignant lesions ranged from $0.82 \times 10^{-3} \mathrm{~mm}^{2} /$ seconds to $1.65 \times 10^{-3} \mathrm{~mm}^{2} /$ seconds, with a mean $A D C$ value of $1.14 \pm 0.22 \times 10^{-3} \mathrm{~mm}^{2} /$ seconds. In benign lesions, the mean ADC was $1.62 \pm 0.24 \times 10^{-3} \mathrm{~mm}^{2} /$ seconds, varying from $1.17 \times 10^{-3} \mathrm{~mm}^{2} /$ seconds to $2.00 \times 10^{-3} \mathrm{~mm}^{2} /$ seconds.
Conclusions The preliminary results show that contrast-enhancement patterns are mostly aspecific, while there is a better concordance between tumour malignancy and ADC values. Inclusion of more patients in the future might result in a threshold value of ADC allowing malignant lesions to be distinguished from benign lesions.

\section{P17}

Phase I study of combination therapy with weekly paclitaxel and cyclophosphatamide for advanced or recurrent breast cancer

T Nakayama1', N Masuda2', J Yamamura², S Kamigaki³, T Taguchi', M Hatta4, J Sakamoto ${ }^{4}$

${ }^{1}$ Graduate School of Medicine, Department of Breast and Endocrine Surgery, Osaka University, Osaka, Japan; ${ }^{2}$ Department of Surgery, Breast Oncology Group, Osaka National Hospital, Osaka, Japan; ${ }^{3}$ Department of Surgery, Sakai Municipal Hospital, Osaka, Japan; "4Graduate School of Medicine, Social Life Science, Young Leaders' Program, Nagoya University, Aichi, Japan

Breast Cancer Research 2009, 11(Suppl 1):P17 (doi: 10.1186/bcr2300) Objective Although anthracycline is a key agent in breast cancer treatment, there is a concern that it may cause cardiotoxicity. Recently, the usefulness of combined therapy with docetaxel and cyclophosphamide (C) was reported. Because paclitaxel (P) has different features, such as induction of apoptosis and anti-angiogenic activity on weekly administration, establishment of combination therapy of $P / C$ is required. We initiated a phase I study to determine the maximum tolerated dose and the recommended dose (RD) of the combination therapy of $P / C$ for advanced or recurrent breast cancer.

Methods $P$ was given intravenously on days 1,8 and 15 and $C$ on day 1 , every 3 weeks. $P$ was given at $80 \mathrm{mg} / \mathrm{m}^{2}$ for level 1 and $100 \mathrm{mg} / \mathrm{m}^{2}$ for level 2 , and $\mathrm{C}$ at $600 \mathrm{mg} / \mathrm{m}^{2}$ for both. Onset of dose-limiting toxicity was evaluated during course 1 , and tolerability through course 4 .

Results Four patients each were enrolled in levels 1 and 2 from October 2006 to November 2007. Main adverse events were four cases of grade 3 neutropenia (50\%) and one case of peripheral nerve disorder (12.5\%). During the first course of levels 1 and 2, hematologic toxicity of grade 4 and nonhematologic toxicity of grade 3 or higher were not observed, and a MTD was not attained. The response rate among assessable cases (one in level 1, two in level 2) was 66.7\%.

Conclusions Safety was confirmed during four courses at level 2, and this was regarded as the RD. This is the first report on the phase I study of the combination therapy of weekly $\mathrm{P}$ and $\mathrm{C}$, and its safety and efficacy should be evaluated in the phase II trial.

\section{P18 \\ Role of the BRCA1 gene in stem cells and treatment of mammary gland cancer \\ H Rassi}

Molecular Diagnosis Center, Jam Aria, Tehran, Iran

Breast Cancer Research 2009, 11(Suppl 1):P18 (doi: 10.1186/bcr2301)

The mammary gland is a dynamic organ that undergoes significant developmental changes during pregnancy, lactation, and involution. Mammary gland cancer may develop through dysregulation of selfrenewal pathways of normal mammary stem cells. The regulation of the self-renewal, differentiation, and migration of mammary stem cells and their progenitors that are localized in the mammary glands appears to be assumed through distinct developmental signalling pathways such as hormones, EGF, hedgehog, Wnt//-catenin, Notch, and Bmi-1. Several tumour suppressors inhibit regenerative capacity by promoting cell death or senescence in stem cells. The breast cancer susceptibility gene $(B R C A 1)$ is a tumour suppressor gene expressed in many nuclear processes, including stem cell regulation, DNA damage repair, recombination, transcription, ubiquitination, cell cycle checkpoint enforcement, and centrosome regulation. BRCA1 is mutated in about one-half of all hereditary mammary gland cancer cases, and its 
expression is frequently decreased in sporadic cancers. Women with hereditary mammary gland and ovarian cancer due to BRCA1 mutations are born with a mutation in one BRCA1 allele, but only develop cancer after mutation or allelic loss of the other $B R C A 1$ allele. $B R C A 1$ plays a critical role in the differentiation of ER-negative stem/progenitor cells from ER-positive luminal cells. Defects of the $B R C A 1$ gene may result in the accumulation of genetically unstable mammary gland stem cells, providing prime targets for further carcinogenic events, because $B R C A 1$ also plays a role in DNA repair. Our study indicates that restoration of normal wild-type BRCA1 expression levels in many mammary gland cancers may inhibit tumours by a genetic correction strategy, wherein the loss of $B R C A 1$ expression contributes to tumorigenesis.

\section{P19}

\section{Advanced topics in array comparative genomic hybridization for diagnosis of hereditary breast cancer H Rassi}

Molecular Diagnosis Center, Jam Aria,Tehran, Iran

Breast Cancer Research 2009, 11(Suppl 1):P19 (doi: 10.1186/bcr2302)

Array comparative genomic hybridization $(\mathrm{aCGH})$ is an efficient approach for scanning entire genomes to seek variations in genomic copy number variations, genotyping and medical genetics. It is changing from being only a research tool to being a tool for clinical diagnostics in patients with cancer, global developmental delay, mental retardation, autism, multiple congenital anomalies and dysmorphism, and is becoming a powerful tool in disease gene discovery and prenatal diagnostics. This tool is also showing promising data in cancer research and in the diagnosis, classification and prognosis of hereditary breast cancer. This presentation discusses the techniques available for aCGH analysis and possible approaches for using aCGH as a tool for the identification of hereditary breast cancer.

\section{P20}

\section{Advanced topics in BRCA1/BRCA2 analysis by genetic analyzer and real-time PCR \\ H Rassi}

Molecular Diagnosis Center, Jam Aria, Tehran, Iran

Breast Cancer Research 2009, 11(Suppl 1):P20 (doi: 10.1186/bcr2303)

The most common gene changes in breast cancer are those of the $B R C A 1 / B R C A 2$ genes. As both are large multi-exon genes, mutation screening of $B R C A 1 / B R C A 2$ is technically challenging, because each gene harbors more than 1,000 different disease-associated mutations, the vast majority of which are individually rare. However, methods widely used in research laboratories such as SSCP, DGCG and HPLC miss nearly one-third of the BRCA mutations that are detected by genetic analyzer. These methods for scanning mutation fail to detect all $B R C A 1$ germline defects. In the meantime, an alternative approach is to use a mutation scanning technique to highlight variations in genomic sequences that are then characterized by genetic analyzer. On the other hand, large genomic rearrangements are not detectable by current PCR-based methods, perhaps explaining the discrepancy between linkage analysis and genetic testing. Quantitative real-time PCR can determine large genomic rearrangements, gene duplications or deletions in BRCA1/BRCA2 genes. Furthermore, melting curve analysis immediately after PCR can identify small mutations, down to single base changes. These techniques are becoming easier and faster and can be multiplexed. In summary, sequencing in combination with real-time PCR methods is a favorable option for the analysis of $B R C A$

\section{P21}

\section{Breast cancer survivors and lymphoedema: the} relevance of education

E Uña1', G Fernández², A Ceballos ${ }^{3}$, E Hortelano ${ }^{3}$, C Perea $^{3}$, F López-Lara ${ }^{3}$

${ }^{1}$ Servicio de Oncología Médica, Hospital Clínico Universitario de Valladolid, Spain; ${ }^{2}$ Servicio de Oncología Radioterápica, Hospital Central de Asturias de Oviedo, Spain; ${ }^{3}$ Servicio de Oncología Radioterápica, Hospital Clínico Universitario de Valladolid, Spain Breast Cancer Research 2009, 11(Suppl 1):P21 (doi: 10.1186/bcr2304) Introduction Secondary arm lymphoedema is a chronic and distressing condition, relatively common after axillary lymph node dissection (AND) for breast cancer. It may be associated with functional, esthetic, and psychological problems that actually could affect the quality-of-life (OOL) of breast cancer survivors. The present study describes the prevalence and characteristics of arm and hand swelling in patients undergoing breast cancer surgery in our institution.

Methods We studied patients who underwent AND for breast cancer who had been diagnosed 4 years ago. We analyzed the prevalence of lymphoedema in these patients and reported data about pathologic and surgical characteristics.

Results Seventy-five women, median age 61 years, were included. Nine women (12\%) reported arm or hand swelling since their surgery, eight of them with mild swelling. Twenty-one (28\%) reported current swelling and one-half of them constant swelling, mainly in the hand. Swelling was considered mild when it affected just the hand. Women who reported severe swelling had significantly worse physical functioning and depressive symptoms as well. Since the swelling has reduced the possibility of dressing appropriately and modified perceptions about general appearance, $92 \%$ of these women began treatment for swelling with a high grade of compliance (86\%). We detected that just five women had high body mass index; all of them had more than 10 axillary lymph nodes evaluated and one-half of them were affected by the tumor.

Conclusions Arm lymphoedema is a chronic problem for a subgroup of long-term survivors of breast cancer that negatively affects physical functioning. Educational efforts are needed for being aware of this problem so that survivors could identify this complication early and begin treatment to improve their physical functioning.

\section{P22}

Sentinel lymph node biopsy for breast cancer: how many nodes to stop at? Experience at a tertiary centre in Singapore SYJ Pang1, WS Yong' ${ }^{2}$ CY Wong', GH Ho², G Chan'

${ }^{1}$ Department of General Surgery, Singapore General Hospital, Singapore; ${ }^{2}$ Department of Surgical Oncology, National Cancer Centre, Singapore

Breast Cancer Research 2009, 11(Suppl 1):P22 (doi: 10.1186/bcr2305)

Objective Sentinel lymph node biopsy (SLN) using blue dye and radioisotope is a widely used tool in the staging of breast cancer. However, multiple SLNs are frequently found. We sought to determine whether there is safe number of SLNs at which to stop the procedure without affecting accuracy.

Methods We reviewed the records of 182 patients who underwent successful SLN biopsy using radioisotope and/or blue dye in Singapore General Hospital. SLNs with counts more than $10 \%$ of the background axilla count, with retention of blue dye or SLNs that were enlarged were removed and the order of removal recorded. All SLN sites removed were categorized as a dye success, an isotope success or both. The count for each SLN site (if applicable) was recorded.

Results Seventy-nine percent of the patients who underwent SLN biopsy (144) had multiple SLNs. The mean number of SLNs removed per patient was 2.4. Twenty-six percent of the patients (48) were nodepositive. Eighty-five percent of node-positive patients (41) had multiple 
SLNs. Eighty-eight percent of the node-positive patients were identified within the first two sites, while $97.5 \%$ and $100 \%$ were identified by the third and fourth sites, respectively.

Conclusions Seventy-nine percent of patients undergoing SLN biopsy for breast cancer have multiple SLNs. Positive nodes were detected within the first three sites removed in $97.5 \%$ of node-positive patients. Terminating the procedure at the third SLN site may help in lowering morbidity and costs without compromising accuracy.

\section{P23}

Sentinel node in breast cancer: retrospective analysis of $\mathbf{2 1 2}$ patients and factors associated with lymphatic involvement A López López1, S Ruiz Solís', R De Juan Rubio1, CM Hentea1,
D Lora Pablos², A Gómez Embuena', A Manrique Legaz', MJ Tabuenca Mateo ${ }^{1}$

${ }^{1}$ Nuclear Medicine Department, and ${ }^{2}$ Research Unit, Hospital 12 de Octubre, Madrid, Spain

Breast Cancer Research 2009, 11(Suppl 1):P23 (doi: 10.1186/bcr2306)

Objective To perform a retrospective study/review of the sentinel lymph node biopsy (SLNB) technique at our institution, analysing (from the results obtained) the relationship with the patients' age, tumour characteristics, injection technique, lymph node involvement and local/regional recurrence.

Materials and methods A total of 212 patients were included; 209 patients underwent subdermal periareolar injection (of $99 \mathrm{mTc}$ nanocolloid) and peritumoral injection was performed in three patients. Lymphoscintigraphy was performed after 15 to 30 minutes postinjection until the sentinel lymph nodes (SLN) were identified, and anterior and lateral views were registered. In the case of a next-day surgery protocol, a delayed 24-hour post-injection view was registered preoperatively. Skin marking of the SLN location was performed preoperatively. SLN were defined as the ones presenting with the highest count rate as measured by the intraoperative probe, as well as those with count rate of at least $>10 \%$ of the former. The remaining resected LN were regarded as non-SLN (NSLN). A descriptive (and association) analysis of the following variables was performed: age, size and tumour location, histology type and grade, injection technique, number of removed SLN and NSLN, malignant involvement of SLN and NSLN, surgical axillary clearance (AC), malignant lymphadenopathy in $\mathrm{AC}$ and axillary recurrence.

Results The mean patient age was 59 (range 32 to 85 ) years; mean of removed SLN 2.94; mean of removed NSLN 0.82. The primary tumour was palpable in 115 patients (54.25\%). The most frequent location was the supero-external quadrant $(45.75 \%)$. The size of most tumours was between 1 and $2 \mathrm{~cm}$ (34.43\%). The most common histological group was ductal carcinoma (60.38\%) and the most common histology grade was 2 (37.74\%). In $91.08 \%$ of cases, the number of removed SLN was $\leq 5$. SLN were positive in 49 patients (23.11\%). In $92.45 \%$, $\geq 3 \mathrm{NSLN}$ were removed. In six patients $(2.83 \%)$ the NSLN were positive on histology, and in three of them SLN were negative. There was a statistically significant relationship between tumour size and malignant involvement of the SLN and NSLN, the indication of AC and the presence of malignant lymphadenopathy in AC. Moreover, there was a statistically significant relationship between histology grade and the number of removed SLN.

Conclusions The local and regional lymph node involvement of disease only showed a statistically significant relationship with tumour size. The number of removed SLN showed a statistically significant relationship with histology grade. It would be advisable to perform a histological analysis of all removed SLN and NSLN.
P24

Efficacy and safety of the administration of bevacizumab in combination with first-line chemotherapy for the treatment of advanced breast carcinoma: ATHENA MO19391 study results in Spanish patients

EM Ciruelos', G López-Vivanco², I Peláez ${ }^{3}$, J Rifa4, A Santaballa ${ }^{5}$, S González ${ }^{6}$, MA Segui ${ }^{7}$, C Jara $^{8}$, I Alvarez ${ }^{9}$, H Cortés-Funes ${ }^{1}$

${ }^{1}$ Hospital Universitario 12 de Octubre, Madrid, Spain; ${ }^{2}$ Hospital de Cruces, Baracaldo Vizcaya, Spain; ${ }^{3}$ Hospital de Cabueñes, Gijón, Asturias, Spain; ${ }^{4}$ Hospital Son Dureta, Palma de Mallorca, Spain; ${ }^{5}$ Hospital Universitario la Fe, Valencia, Spain; ${ }^{6}$ Mutua de Terrasa, Barcelona, Spain; ${ }^{7}$ C.S. Parc Taulí, Sabadell, Barcelona, Spain; ${ }^{8}$ F. Hospital de Alcorcón, Madrid, Spain; ${ }^{9}$ Hospital de Donosti, San Sebastián, Spain

Breast Cancer Research 2009, 11(Suppl 1):P24 (doi: 10.1186/bcr2307) Introduction Bevacizumab (BVZ) is a humanized monoclonal antibody that targets vascular endothelial growth factor, that leads to the inhibition of growth and proliferation of new blood vessels that are next to the tumor. The administration of BVZ plus first-line chemotherapy (paclitaxel, docetaxel) in the treatment of advanced breast carcinoma has lead to better outcomes in terms of response rate and time to progression in previous published studies.

Methods The ATHENA MO19391 study is a nonrandomized, openlabeled, international trial that tests the addition of BVZ $(10 \mathrm{mg} / \mathrm{kg} /$ 2 weeks or $15 \mathrm{mg} / \mathrm{kg} / 3$ week) to first-line chemotherapy (nonanthracycline based) in the treatment of advanced breast cancer. Results of the ATHENA trial have been previously reported. In the global study, 2,027 patients were included in 37 participating countries, between September 2006 and June 2008. This report shows the current efficacy and safety data of ATHENA participating patients included in Spanish centers.

Results A total of 119 patients in 20 Spanish centers were included in the trial, with the following basal characteristics: median age 51 years (27 to 79); postmenopausal status, 83 patients (69.7\%); estrogen receptor-positive, 64 patients (66.7\%); HER2-negative, HER2-positive, unknown, 92 patients $(95.8 \%)$, two patients $(2.1 \%)$, two patients (2.1\%), respectively; prior adjuvant therapy, 92 patients $(95.8 \%)$ anthracycline-based, 63 patients $(72.4 \%)$ and taxane-based, 38 patients $(43.6 \%)$. The majority of patients (68 patients, $57.1 \%$ ) who received previous adjuvant treatment had a disease-free interval $>24$ months.

The most common chemotherapy regimens that were combined with BVZ were paclitaxel (48 patients, $40.3 \%$ ) and docetaxel (41 patients, $34.5 \%$ ). Other used regimens were paclitaxel + gemcitabine (six patients, 5\%), capecitabine (three patients, $2.5 \%$ ), and others. The median number of administered cycles per patient was 8 ( 1 to 27). With a median follow-up of 10.9 months (0.9 to 24.9 ), $46 \%$ of patients had disease progression. The median time to progression was 11.5 months (10 to 13.6), and median overall survival was 22.5 months (19.2 to NR). One hundred and ten patients were evaluable for efficacy. Fourteen patients (12.7\%) achieved a complete response, and 60 patients $(54.5 \%)$ a partial response, for an overall response rate of $67.2 \%$. Twenty-seven patients $(24.5 \%)$ had stable disease, and nine $(8.2 \%)$ progressed. The clinical benefit rate was $91.7 \%$. Thirty-one patients $(26.1 \%)$ had at least one adverse event grade $\geq 3$. The most relevant SAEs were: febrile neutropenia, five patients (4.2\%); grade 1 to 2 hypertension, 41 patients $(34.4 \%)$; grade 3 to 4 hypertension, eight patients $(6.7 \%)$; grade 1 to 2 epistaxis, 60 patients $(50.4 \%)$; grade 3 DVT, one patient $(0.8 \%)$; grade 1 to 3 proteinuria, 32 patients $(26.9 \%)$; grade 4 nephrotic syndrome, two patients $(1.7 \%)$; grade $3 \mathrm{GI}$ perforation, one patient $(0.8 \%)$; and grade 1 to 2 impaired healing, four patients (3.4\%). Thirty-two patients $(26.9 \%)$ had died at the time of the analysis, the majority (31 patients) due to breast cancer progression. Conclusions Chemotherapy (nonanthracycline based) plus BVZ as first-line treatment for advanced breast cancer has a high antitumor 
activity in terms of response rate and clinical benefit and of time to progression. The toxicity profile is manageable and due to toxic effects of both treatments separately. Administration of BVZ plus chemotherapy must be considered first-line therapy for the treatment of advanced breast cancer.

\section{P25}

Circulating epithelial tumor cells in patients with metastatic breast cancer treated with bevacizumab L Manso, E Ciruelos, A Rodríguez, J Diaz, C Mendiola, JA López-Martín, H Cortes-Funes

Hospital 12 de Octubre, Madrid, Spain

Breast Cancer Research 2009, 11(Suppl 1):P25 (doi: 10.1186/bcr2308)

Introduction Circulating epithelial tumor cells (CTCs) in peripheral blood are an ideal source for the detection of disseminated tumor cells of an easy sampling procedure. Their prognostic significance has been demonstrated in metastatic breast carcinoma and has the potential to influence the clinical management of patients with breast cancer. The aims of the present study are the evaluation of the prevalence and kinetics of CTS before and after anti-angiogenic treatment with bevacizumab (Avastin) in patients with metastatic breast cancer.

Methods We analyzed $7.5 \mathrm{ml}$ peripheral blood from 40 metastatic breast cancer patients treated with bevacizumab before (baseline) and after the first cycle of treatment. The presence of CTCs was assessed with the CellSearch System (Veridex, USA). Samples were subjected to immunomagnetic enrichment with an anti-EpCAM antibody and were fluorescence labeled. CTCs were defined as nucleated cells (DAPI $\left.{ }^{+}\right)$ expressing cytokeratin 8, 18 and 19 but CD45-negative phenotype. A sample was considered positive when one or more cells were detected.

Results Data are available for 40 patients. We found $\geq 1$ CTCs before the first cycle of treatment with bevacizumab in $60 \%$ of the patients $(n=24)$. After the first treatment, reduction of one or more CTCs was found in $35 \%$ of the patients $(n=14)$. The median number of CTCs was 15 cells $/ 7.5 \mathrm{ml}$ blood in the first determination and 8 cells $/ 7.5 \mathrm{ml}$ in the second determination. In $30 \%$ of the patients $(n=12)$ we found an increase of baseline CTCs before and after treatment. In 26 patients we did not found any variation of baseline CTCs before and after treatment. In eight patients with CTCs positive at baseline (20\%) the second determination after treatment was 0 cells $/ 7.5 \mathrm{ml}$. Persistence of at least one CTC after the first cycle of treatment was found in $35 \%$ of the patients.

Conclusions The results of this explorative study are preliminary and a large number of patients and follow-up are required. The study is ongoing to explore the prognostic significance of reduction or persistency of CTCs during treatment with anti-angiogenic agents (bevacizumab) and the relationship with response rate to treatment. Complete data will be presented.

\section{PROFFERED PAPERS}

\section{P26}

\section{Clinical features and prognosis of triple-negative breast cancer}

M Izquierdo Sanz', A Alsina Maqueda', M Cabero Riera', R Fabregas Xaurado', F Tresserra Casas ${ }^{2}$, M Cusido Gimferrer ${ }^{1}$, C Ara Perez ${ }^{1}$, A Úbeda Hernandez ${ }^{1}$

${ }^{1}$ Department of Obstetrics, Gynecology and Reproductive Medicine, and Institut Universitari Dexeus, Barcelona, Spain; ${ }^{2}$ Department Histology, Institut Universitari Dexeus, Barcelona, Spain

Breast Cancer Research 2009, 11(Suppl 1):P26 (doi: 10.1186/bcr2309) Objective To compare the clinical features and prognosis of triple-
Methods Analysis of all breast cancers studied by the Breast Diseases Committee during the period 2000 to 2005, comparing the clinical features and prognosis of triple-negative with the rest of breast cancers. The overall survival, local recurrence and contralateral breast cancer were analyzed with Kaplan-Meier curves.

Results We studied 345 breast cancers, 22 (6.4\%) triple negative and $323(93.6 \%)$ nontriple negative. In the triple negative, the tumour size was pT0 0 (0\%), pT1a 1 (7.1\%), pT1b 1 (7.1\%), pT1c 8 (57.1\%), pT2 $3(21.4 \%)$, pT4b $1(7.1 \%)$; and the axillary lymph node was pNO 9 (64.3\%), pN1 5 (35.7\%), with no statistically significant differences for the nontriple negative. The histological grade was grade III in $52.9 \%$ of the cases of triple-negative breast cancer and in 13.8\% of the cases of nontriple-negative breast cancer; these differences were statistically significant. The overall survival was statistically worse, the local recurrences and contralateral breast cancer were higher in triple negative.

Conclusions Triple-negative breast cancer has a high histological grade, more metastases, more local recurrences and contralateral breast cancer, and worse overall survival.

\section{P27}

Discordance between hormone receptor profile of primary breast cancer and metastatic bone disease: should bone marrow biopsy be considered a standard of care?

E Amir ${ }^{1}$, R Broom 1 , O Freedman'1, WS Ooi' ${ }^{1}$, S Done ${ }^{2}$, D Gianfelice ${ }^{3}$, D Barth ${ }^{1}$, H Kahn ${ }^{4}$, M Clemons ${ }^{1}$

${ }^{1}$ Division of Medical Oncology and Hematology, Princess Margaret Hospital, Toronto, Canada; ${ }^{2}$ Department of Pathology, University Health Network, Toronto, Canada; ${ }^{3}$ Department of Interventional Radiology, University Health Network, Toronto, Canada; ${ }^{4}$ Department of Pathology, Sunnybrook Hospital, Toronto, Canada

Breast Cancer Research 2009, 11(Suppl 1):P27 (doi: 10.1186/bcr2310)

Objective The treatment of bone metastases in breast cancer patients is based on the hormone receptor status of the primary tumour. Discordant receptor expression between primary and metastatic tumours has been reported in around 20 to $60 \%$ of cases. The present study, therefore, aimed to prospectively explore the incidence of discordant receptor status in primary and metastatic bone disease, and to evaluate the role of bone marrow biopsies for the reassessment of receptor status.

Methods Nineteen patients with known bone metastases underwent both a CT-guided bone metastasis biopsy as well as bone marrow aspirate and trephine. The estrogen receptor (ER) and progesterone receptor (PR) of these samples was assessed and compared with those of primary breast cancer.

Results Tumor cells were found in $13(68.4 \%)$ bone metastasis samples and in nine (47.4\%) bone marrow biopsies. Discordance between the primary and metastatic samples was seen in 10 patients (52.6\%). Among these, ER and PR changed from positive to negative in seven cases and from negative to positive in one case. In six cases $(31.6 \%)$, malignant cells were identified in both bone metastasis and bone marrow samples from the same patient. Among these, ER and PR were concordant in $100 \%$ and $83 \%$ of cases.

Conclusions Given that the receptor profile of metastatic disease is assumed to be the same as the primary tumour, discordance between primary and metastatic cancer can have a significant impact on the outcome of treatment choices. Receptor discordance rates in this analysis were similar to that which has been reported in previous studies. There appeared to be good concordance between bone metastasis and bone marrow biopsies. Bone marrow biopsy may therefore be a simple, safe and well-tolerated way to obtain tissue to reassess receptor status of metastatic breast cancer, and should be considered before the more invasive bone metastasis biopsy. 


\section{P28}

ZD6474 in combination with paclitaxel and UVB irradiation enhances the antiproliferative effect and apoptosis on breast carcinoma

\section{S Sarkar, M Mandal}

School of Medical Science and Technology, Indian Institute of Technology, Kharagpur, India

Breast Cancer Research 2009, 11(Suppl 1):P28 (doi: 10.1186/bcr2311) Breast cancer is the leader of cancer deaths among women. Despite developments in surgery, chemotherapy and radiotherapy, the longterm survival of patients with metastatic and higher staging breast cancer has remained very low. Breast cancers and their aggressive nature are characterized by overexpression of epidermal growth factor receptor (EGFR) and vascular endothelial growth factor receptor (VEGFR). There is a common downstream signaling molecule involved in both EGFR and VEGFR mediated pathways. There are reports that cancer cells acquired resistance to anti-EGF/EGFR therapy by increased tumor-induced angiogenesis due to the constitutive overexpression of VEGF. Hence, the inhibition of both EGFR and VEGFR signaling pathways simultaneously may provide greater benefit than blockade of either pathway alone. There are also reports that breast cancer acquired resistance to conventional chemotherapy and radiotherapy by increased phosphorylation of EGFR and VEGFR. Moreover, patients suffering from breast cancer have a poor prognosis because of a lack of effective treatment strategies. We hypothesized that inhibiting the phosphorylation of the EGFR and VEGFR by ZD6474, a dual tyrosine kinase inhibitor of EGFR and VEGFR, in combination with paclitaxel or radiation would inhibit breast cancer cell growth. We tested this hypothesis using the human breast cancer cell lines MCF-7 and MDA-MB-231. In culture, ZD6474 in combination with paclitaxel or UVB had a synergistic effect in inhibition of cell proliferation, and lowered the $\mathrm{IC}_{50}$ by 10 -fold and fivefold, respectively, compared with paclitaxel/UVB alone. Flow cytometry data suggest that combination therapy of ZD6474 with paclitaxel increases apoptosis from $20 \%$ to $45 \%$ in MDA-MB-231 and from $16 \%$ to $38 \%$ in MCF-7 compared with paclitaxel alone. This is further supported by a decrease in cell cycle regulatory protein cyclin $D_{1}$ and an increase in apoptosis marker poly(ADP-ribose) polymerase in combination therapy compared with paclitaxel and UVB irradiation alone. Moreover, combination therapy sensitizes the paclitaxel resistance MCF-7/PTX and MDA-MB231/PTX. ZD6474 along with paclitaxel or radiation can therefore be used for treatment in drug-insensitive advanced breast cancers.

\section{P29}

Developing a prediction model for benefit from fulvestrant in heavily pretreated metastatic breast cancer patients

E Amir ${ }^{1}$, OC Freedman', S Chia ${ }^{2}$, T Petrella ${ }^{3}$, S Dent ${ }^{4}$, K Tonkin', I Ahmad ${ }^{6}$, D Rayson ${ }^{7}$, G Dranitsaris', M Clemons ${ }^{1}$

${ }^{1}$ Division of Medical Oncology and Hematology, Princess Margaret Hospital, Toronto, Canada; ${ }^{2}$ Division of Medical Oncology, British Columbia Cancer Agency, Vancouver, Canada; ${ }^{3}$ Division of Medical Oncology, Sunnybrook Hospital, Toronto, Canada; ${ }^{4}$ Division of Medical Oncology, Ottawa General Hospital, Ottawa, Canada; ${ }^{5}$ Division of Medical Oncology, Cross Cancer Institute, Edmonton, Canada; ${ }^{6}$ Division of Medical Oncology, Saskatoon Cancer Centre, Saskatoon, Canada; ${ }^{7}$ Division of Medical Oncology, Queen Elizabeth II Health Sciences Centre, Halifax, Canada

Breast Cancer Research 2009, 11(Suppl 1):P29 (doi: 10.1186/bcr2312) Introduction Fulvestrant use in heavily pretreated patients with metastatic breast cancer is associated with highly variable responses. The present study aimed to characterize the benefit of fulvestrant therapy and to develop a prediction model for clinical benefit in this setting.

Methods A nationwide, retrospective chart review of patients enrolled in a Canadian compassionate use program was performed. This program mandated prior therapy with tamoxifen and both steroidal and nonsteroidal aromatase inhibitors. Charts from the seven highest accruing centers were reviewed. The sample size was based on the derivation of a model to predict the probability of a patient remaining on fulvestrant and free from chemotherapy for at least 3 months.

Results A total of 305 women received at least one dose of fulvestrant; 207 went on to receive chemotherapy (68\%). Of these, 48 $(23 \%)$ required chemotherapy at 3 months, $113(55 \%)$ at 6 months, and $170(82 \%)$ by 12 months. The median duration of fulvestrant treatment was 126 days (range 23 to 1,920 ). The median overall survival from start of fulvestrant was 698 days (25th percentile, 316 days to 75 th percentile, 1,359 days). The preliminary prediction model showed that older age $(\mathrm{OR}=0.96,95 \% \mathrm{Cl}=0.93$ to 0.99$)$ and having received no adjuvant hormonal therapy $(\mathrm{OR}=0.5,95 \% \mathrm{Cl}=$ 0.2 to 1.25$)$ predicted a greater chance of remaining chemotherapyfree at 3 months. Presence of lung (OR $=2.55,95 \% \mathrm{Cl}=1.1$ to 5.9 ) or brain metastases (OR $=12.8,95 \% \mathrm{Cl}=4.1$ to 55.4) predicted a lower chance of remaining chemotherapy-free at 3 months.

Conclusions Older age and having received no prior adjuvant hormonal therapy predicted a greater chance of remaining chemotherapy-free at 3 months, while lung and brain metastases predicted a lower chance. These factors may be considered when prescribing fulvestrant.

\section{P30}

Employing multiplex immunofluorescence to quantify Her2 and phosphorylated Her2 in formalin-fixed, paraffin-embedded breast tumor specimens MJ Donovan 1,2, P Puig'2, N Erill2, M Clayton', S Hamann', F Khan', D Powell', J Costa 1,2,3, C Cordon-Cardo1,2,4, J Baselga ${ }^{5}$ ${ }^{1}$ Aureon Laboratories, Yonkers, NY, USA; 2 Althia, Barcelona, Spain; ${ }^{3}$ Yale University School of Medicine, New Haven, CT, USA; ${ }^{4}$ Columbia University, New York, NY, USA; ${ }^{5}$ Vall d'Hebron University Hospital, Barcelona, Spain

Breast Cancer Research 2009, 11(Suppl 1):P30 (doi: 10.1186/bcr2313) Objective Her2 overexpression is a predictor for response to trastuzumab; however, only one-third of patients initially respond and the majority progress within 1 year. Recently, levels of the phosphorylated form of Her2 (pHer2) in hormone receptor-positive, primary tumors was determined to be an independent predictor for poor disease-free and overall survival, suggesting that additional variables may be important for determining outcome. We sought to develop a multiplex immunofluorescent (IF) quantitative assay for Her2 that included pHer2 and to compare the initial results with traditional methods of evaluating Her2 levels in formalin-fixed, paraffin-embedded (FFPE) breast tissue specimens.

Methods We developed a Her2 multiplex IF assay (Her2mplex) composed of AE1/AE3 (cytokeratin), TAB250 (Her2-ECD), c-erb B2 (Her2), and Her2 pY1248 (pHer2) utilizing breast tumor cell lines (BTC: MCF7, T47D and SKBR3) that differentially express Her2 and pHer2. The assay was applied to a 37-patient tissue microarray (TMA) with known Her2 status (imunohistochemical (IHC) HercepTest). Image analysis generated quantitative IF intensity features that were evaluated univariately, and then multivariately with feature selection to discriminate Her2 (2+) from Her2 (3+). The AUC was used to estimate performance.

Results We developed baseline intensity thresholds from the component biomarkers in the Her2mplex (TAB250 Her2, c-erb B2 and phosphorylated Her2) utilizing a series of BTC: MCF7, T47D and SKBR3. IF images were acquired from 72 TMA cores, and 52 cores (26 patients) had appropriate tumor content $(>50 \%)$ and quality for analysis. Both c-erB B2 and pHer2 were significantly associated with $\mathrm{IHC}$ data and were selected (AUC $=0.96$, sensitivity $=0.91$, specificity $=1$ ) to discriminate Her2 $(2+)$ from Her2 $(3+)$.

Conclusions Quantitation of Her2 and pHer2 by IF is feasible in FFPE breast tumor specimens. The results correlate with IHC protein data and also provide a quantitative strategy for the development of a standardized assay useful for tumor phenotyping, patient stratification and therapeutic indication. 


\section{P31}

Biopsy confirmation of metastatic breast cancer: interim results of a prospective biopsy study E Amir ${ }^{1}$, O Freedman', C Simmons', N Miller', W Geddie'2, A DeBorja', H Maung', D Gianfelice $^{3}$, K Murphy' ${ }^{3}$, M Clemons'

${ }^{1}$ Division of Medical Oncology, Princess Margaret Hospital, Toronto, Canada; ${ }^{2}$ Department of Pathology, University Health Network, Toronto, Canada; ${ }^{3}$ Department of Interventional Radiology, University Health Network, Toronto, Canada

Breast Cancer Research 2009, 11(Suppl 1):P31 (doi: 10.1186/bcr2314)

Objective Decisions about systemic treatment of women with metastatic breast cancer are often based on oestrogen receptor (ER), progesterone receptor $(\mathrm{PgR})$, and HER2/neu status of the primary tumour. The present study prospectively investigated concordance in receptor status between primary tumour and distant metastases at various stages of progression and assessed the impact of any discordance on patient management.

Methods Biopsies of suspected metastatic lesions were obtained from patients and were analysed for ER/PgR and HER2/neu. The receptor status of metastases was compared with the primary tumour. The treating physician completed questionnaires before and after biopsy to determine whether the biopsy resulted in a change to the treatment plan.

Results Eighty women were enrolled and 70 of them underwent biopsy. Of these, $43(61 \%)$ were newly diagnosed with metastatic disease, $10(14 \%)$ had received one line of treatment in the metastatic setting and $17(24 \%)$ had received two or more lines of treatment in the metastatic setting. Fifty-six samples were sufficient for analysis; of these, 4/56 biopsies (7\%) showed benign disease and one biopsy (2\%) confirmed a different malignancy (idolent lymphoma). Changes in hormone receptor status were observed in 34\% and in HER2/neu status in 5\% of women. Loss of PgR was the most common change in hormone receptor status (29\%). All changes in HER2/neu involved gain of receptor. Biopsy results led to a change of management in 9/70 patients (13\%). Patients with newly diagnosed metastatic disease were more likely to show discordance than those previously treated in the metastatic setting. Triple-negative tumours were least likely to show discordance.

Conclusions This prospective study demonstrates the presence of substantial discordance in receptor status between primary tumour and metastases. The number of patients needed to biopsy to alter management is 7.8. Tissue confirmation should be considered in patients with clinical or radiological suspicion of metastatic recurrence.

\section{P32}

\section{Sentinel lymph node biopsy in breast cancer: day} surgery and local anaesthetic approach F Ricci', T Scala', M Dorkin', E Saralli', LG Capuano', P Picozzi', M Sacchi', R Salvatori'2, A D'Agostini'2, C De Masi³, G Cerimoniale ${ }^{3}$, S Frau $^{3}$, GP Fanelli ${ }^{3}$, R Cianni ${ }^{3}$, A Rossi $^{4}$

${ }^{1}$ Surgery Department, ${ }^{2}$ Nuclear Medicine, ${ }^{3}$ Radiology Department, and ${ }^{4}$ LegaTumori, St M. Goretti Hospital, Latina, Italy

Breast Cancer Research 2009, 11(Suppl 1):P32 (doi: 10.1186/bcr2315) Introduction Sentinel node biopsy (SNB) is widely used in the management of breast cancer patients without axillary metastases. Methods From 1 January 2006 through 31 December 2008 we performed 113 SNBs at St M. Goretti Hospital. Mammary carcinoma was diagnosed as malignant by aspiration cytology and/or biopsy. In all cases with positive cytology, we performed at the same time quadrantectomy and SNB. The patients underwent preoperative lymphoscintigraphy. We never used vital blue dye. All patients underwent surgical treatment 3 to 4 hours later. We performed SNB and quadrantectomy in day surgery (DS) and local anaesthesia (LA). Axillary incision was 2 to $3 \mathrm{~cm}$. This approach was discussed with all patients and informed consent was obtained. The objective of the present study is to investigate the validity of this procedure.
Results Four patients underwent preoperative lymphoscintigraphy, the radiotracer did not show any sentinel lymph node (SLN), and we performed axillary dissection. One case showed a double SLN in the axilla and internal mammary chain. Only the internal mammary node was positive. After surgery we distributed a questionnaire to the patients about the acceptability of this approach. We identified the SLN in all patients (100\%).

Conclusions The results achieved are extremely accurate. This procedure is safe and well accepted by doctors and patients (98\%). The oncological results are absolutely reliable. As regards hospital logistics, operations in DS and LA can be easily managed, leading to a significant cost reduction $-42.15 \%$ less expensive than the same operation performed under general anaesthesia.

\section{P33}

A randomized, phase III trial exploring the effects of neoadjuvant sequential treatment with steroidal (exemestane) and nonsteroidal (anastrozole) aromatase inhibitors on biomarkers in postmenopausal women with hormone receptorpositive locally advanced breast cancer OC Freedman'1, E Amir'1, G Dranitsaris'1, M Dowsett², DEC Cole ${ }^{3}$, H Kahn', F O'Malley ${ }^{5}$, S Verma ${ }^{6}$, M Clemons ${ }^{1}$

${ }^{1}$ Division of Medical Oncology and Hematology, Princess Margaret Hospital, Toronto, Canada; ${ }^{2}$ Department of Biochemistry, Royal Marsden Hospital, London, UK; ${ }^{3}$ Department of Biochemistry, Women's College Hospital, Toronto, Canada; ${ }^{4}$ Department of Pathology, Sunnybrook Hospital, Toronto, Canada; ${ }^{5}$ Department of Pathology, Mount Sinai Hospital, Toronto, Canada; ${ }^{6}$ Division of Medical Oncology, Sunnybrook Hospital, Toronto, Canada

Breast Cancer Research 2009, 11(Suppl 1):P33 (doi: 10.1186/bcr2316) Introduction Despite many large randomised trials assessing adjuvant endocrine treatment for postmenopausal breast cancer patients, the optimal endocrine strategy remains unknown. Neoadjuvant endocrine studies provide the opportunity to model an appropriate study design in a more expeditious manner. Several large adjuvant trials are exploring sequential aromatase inhibitor (Al) strategies. The present study compared the effect of two sequences of Al use - steroidal (exemestane (E)) and nonsteroidal (anastrozole (A)) - on serological and pathological biomarkers, when given in the neoadjuvant setting to patients with locally advanced breast cancer.

Methods Thirty postmenopausal women with estrogen and/or progesterone receptor-positive disease were randomised to receive either $E$ followed by $A$ ( $E \rightarrow A$ group) or $A$ followed by $E(A \rightarrow E$ group). Each agent was given for 8 weeks. Serum estrone sulfate, and estradiol levels, as well as intratumoral Ki67 were evaluated at baseline, 8 weeks, and 16 weeks. Clinical response, patient preference, and quality of life were also assessed.

Results Despite rapid falls in sex steroid levels with Al use, there was no difference in estradiol, estrone sulfate or Ki67 levels between groups. There was no significant difference in toxicities, or in quality of life scores. Overall clinical response rate was $68 \%$ and clinical benefit was $93 \%$. There was a trend towards improved clinical response in the $A \rightarrow E$ group. The majority of patients expressed a preference for treatment.

Conclusions Neither sequence of steroidal or nonsteroidal Al appears to offer a significant advantage over the other. A trend towards improved clinical response in patients treated with $A \rightarrow E$ is hypothesisgenerating and needs confirmation in larger trials. 